Article

\title{
Metabolic Reprogramming during Microglia Activation
}

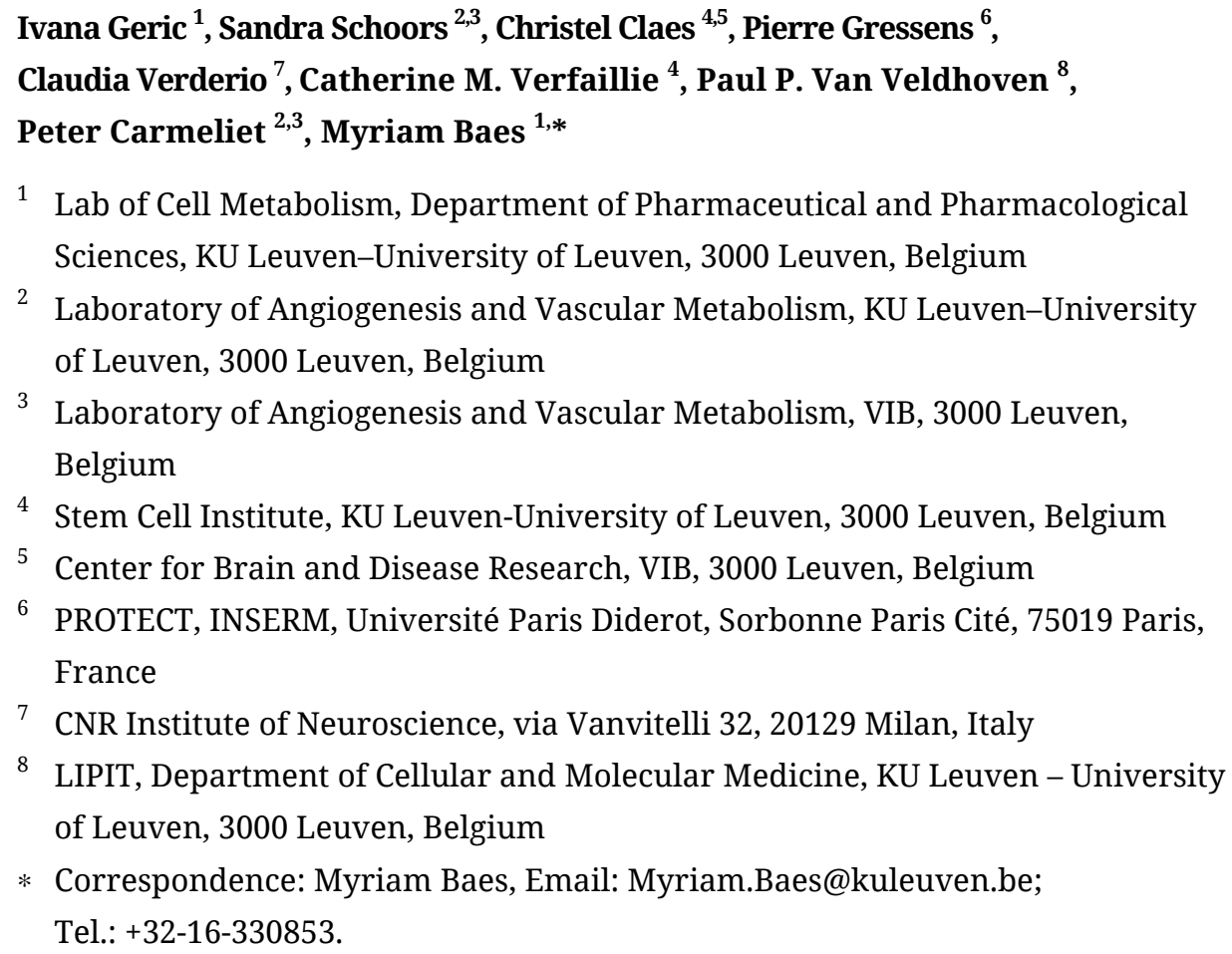

1 Lab of Cell Metabolism, Department of Pharmaceutical and Pharmacological Sciences, KU Leuven-University of Leuven, 3000 Leuven, Belgium

2 Laboratory of Angiogenesis and Vascular Metabolism, KU Leuven-University of Leuven, 3000 Leuven, Belgium

3 Laboratory of Angiogenesis and Vascular Metabolism, VIB, 3000 Leuven, Belgium

4 Stem Cell Institute, KU Leuven-University of Leuven, 3000 Leuven, Belgium

5 Center for Brain and Disease Research, VIB, 3000 Leuven, Belgium

6 PROTECT, INSERM, Université Paris Diderot, Sorbonne Paris Cité, 75019 Paris, France

7 CNR Institute of Neuroscience, via Vanvitelli 32, 20129 Milan, Italy

8 LIPIT, Department of Cellular and Molecular Medicine, KU Leuven - University of Leuven, 3000 Leuven, Belgium

* Correspondence: Myriam Baes, Email: Myriam.Baes@kuleuven.be; Tel.: +32-16-330853.

\begin{abstract}
Microglia, the specialized macrophages of the brain, can adopt different shapes and functions, some of which may be detrimental for nervous tissue. Similar to other immune cells, the metabolic program may determine the phenotypic features of microglia, and could constitute a therapeutic target in neurological diseases. Because the knowledge on microglial metabolism was sparse we here employed mouse primary microglia cells polarized into a pro- or anti-inflammatory state to define their metabolic features. After stimulation with either IL1 $\beta /$ IFN $\gamma$ or IL4, the activity of glycolysis, glucose oxidation, glutamine oxidation, mitochondrial and peroxisomal fatty acid $\beta$-oxidation, and fatty acid synthesis, was assessed by using radiolabeled substrates. We complemented these data with transcriptome analysis of key enzymes orchestrating these metabolic pathways. Pro-inflammatory microglia exhibit increased glucose and glutamine metabolism and suppress both fatty acid oxidation and to a lesser extent fatty acid synthesis. On the other hand, anti-inflammatory microglia display changes only in fatty acid metabolism upregulating both fatty acid oxidation and fatty acid synthesis. Importantly, also human microglia-like cells differentiated from pluripotent stem cells upregulate glycolysis in pro-inflammatory conditions. Finally, we show that glycolytic enzymes are induced in a pro-inflammatory brain environment in vivo in mice. Taken together, the
\end{abstract}


distinct metabolism in pro- and anti-inflammatory microglia can constitute a target to direct the microglial phenotype.

KEYWORDS: microglia; immunometabolism; glycolysis; inflammation

\section{ABBREVIATIONS}

ACOX1, acyl-CoA oxidase 1; AD, Alzheimer's disease; BCFA, branched chain fatty acids; CNS, central nervous system; CPT, carnitine palmitoyltransferase; FAO, fatty acid oxidation; FASN, fatty acid synthase; G6PD, glucose-6-phosphate dehydrogenase; GLUD, glutamate dehydrogenase; GOT, glutamate-oxaloacetate transaminase; GS, glutamine synthase; HK, hexokinase; hPSC, human pluripotent stem cells; IFN, interferon; IL, interleukin; aKG, a-ketoglutarate; LDH, lactate dehydrogenase; LPL, lipoprotein lipase; LPS, lipopolysaccharide; MCAD, medium-chain acyl-CoA dehydrogenase; MFP, multifunctional protein; NO, nitric oxide; OXPHOS, oxidative phosphorylation; PDH, pyruvate dehydrogenase; PDK, pyruvate dehydrogenase kinase; PFK, phosphofructokinase; PFKFB, phosphofructokinase-fructose biphosphatase; PGC, peroxisome proliferator-activated receptor gamma coactivator; PPP, pentose phosphate pathway; PUFA, polyunsaturated fatty acids; ROS, reactive oxygen species; SHPK, sedoheptulokinase; TAG, triacylglycerides; TCA, tricarboxylic acid; VLCFA, very long chain fatty acids

\section{INTRODUCTION}

Microglia, mostly referred to as resident macrophages of the central nervous system (CNS), are responsible for maintaining brain homeostasis through their function that is both glial and immune related [1]. However, unlike most tissue macrophages, they exclusively originate from embryonic progenitors in the yolk sac and are maintained locally throughout development and adulthood [2,3]. Furthermore, microglia possess a unique homeostatic signature distinguishing them clearly from other tissue resident macrophages, but also from the surrounding brain cells, i.e., neurons, astrocytes and oligodendrocytes [4,5]. In a healthy brain, microglia have a ramified morphology, with constantly moving protrusions, surveying the brain tissue [6]. In response to a variety of stimuli or events, microglia can adopt other phenotypes accompanied with specific morphology and function. For example, it has already been shown that treatment of microglia with interleukin (IL)1 $\beta$ or interferon (IFN) $\gamma$ can provoke a pro-inflammatory or so called M1 microglia phenotype, while IL4 can provoke an anti-inflammatory or M2 phenotype [7]. Pro-inflammatory microglia, characterized by an amoeboid shape and production and secretion of inflammatory cytokines and chemokines, were shown to be detrimental and harmful for neuronal tissue [8]. Moreover, the pro-inflammatory cytokine IL1 $\beta$ has been implicated in acute neuroinflammatory conditions and in several 
neurodegenerative diseases [9-11]. In contrast, in an anti-inflammatory state, microglia have protective and beneficial roles with IL4 mediating neuroprotection [8,12-14]. According to current insights, microglia may play a decisive role in the outcome of neurological diseases including ischemia, neurodegeneration and traumatic events. Importantly, chronic activation of immune cells, microglia in particular, presents a common link between these diseases [15].

Manipulating the microglial phenotype has therefore become a promising approach for therapeutic intervention in neurological diseases $[16,17]$. Because the metabolic program of immune cells is not only associated with, but also determinant for their phenotype, this constitutes a potential target. For murine macrophages it was indeed shown that the pro-inflammatory or M1 state is glycolytic while fatty acid oxidation (FAO) and oxidative phosphorylation (OXPHOS) are a hallmark of the anti-inflammatory or M2 state [18,19]. Importantly, glycolysis is indispensable for pro-inflammatory activation of macrophages, since blocking this pathway abrogated the stimulation and secretion of pro-inflammatory cytokines [20]. In a similar manner it was shown that FAO and mitochondrial function are necessary for anti-inflammatory functions [19]. Moreover, approaches to shape the macrophage phenotype via targeting metabolism in chronic inflammatory diseases have already yielded positive results [21]. However, in contrast to the extensive knowledge on metabolism of immune cells in the periphery, not much is known about microglial metabolism. So far, some studies provided evidence indicative of increased glycolysis in pro-inflammatory M1 microglia [22-25]. Furthermore, it has been shown that lipopolysaccharide (LPS)-activated microglia exhibit increased glutamate to glutamine conversion via glutamine synthase (GS) [26,27]. In parallel, LPS-activated microglia exhibit decreased mitochondrial function evidenced by the reduction in the activity of respiratory chain enzymes and the reduction in mitochondrial OXPHOS [22,23,28,29]. However, despite the fact that metabolic alterations in microglia have been implicated in several disease models and in aging [25,30-33], it remains largely unknown how most pathways are regulated. Moreover, metabolic approaches have already been applied in several CNS pathologies and had positive effects, although the cellular target was not defined [31,34-37].

In order to define the metabolism of microglia in various states, we used mouse primary microglia cells polarized into a clear-cut pro- or anti-inflammatory state, through stimulation with IL1 $\beta /$ IFN $\gamma$ or IL4, respectively. We investigated several metabolic pathways, including glycolysis, glucose oxidation, glutamine oxidation, fatty acid $\beta$-oxidation and fatty acid synthesis, by using radiolabeled substrates which allow specific and sensitive measurement of metabolism in the cell. Furthermore, we complemented these data with transcriptome analysis of key enzymes orchestrating these metabolic pathways. Finally, glycolysis was assessed in human microglia-like cells, differentiated from 
pluripotent stem cells. The data indicate dynamic changes in microglial metabolism during polarization.

\section{MATERIALS AND METHODS}

Mice

Mice were bred in the animal housing facility of the KU Leuven, had ad libitum access to water and standard rodent food, and were kept on a $12 \mathrm{~h}$ light and dark cycle. All animal experiments were performed in accordance with the "Guidelines for Care and Use of Experimental Animals" and fully approved by the Research Ethical Committee of the KU Leuven (\#065/2015, 2015-11-03).

\section{Primary Microglia Cultures}

Microglia cells were isolated from the brains of postnatal day 0-1 control C57BL/6J mice, as previously described [7]. Briefly, brains of mice were dissected and meninges were removed under the microscope in sterile conditions. Subsequently, cortices were dissociated and pieces were allowed to sediment for $1 \mathrm{~min}$, after which the supernatant was collected. The supernatant was then centrifuged and pellets were resuspended in DMEM medium supplemented with $10 \%$ fetal bovine serum and $0.01 \%$ penicillin/streptomycin (all from ThermoFisher, Waltham, MA, USA) and divided between T75 culture flasks. Ten T75 flasks were used for plating the cells isolated from 6 brains. After 2 weeks in culture, microglia were separated from astrocytes and collected by reciprocal shaking $(30 \mathrm{~min}$ ) and by repeated rinsing of the flasks, respectively. Cells were plated in 48-, 12- or 6-well plates according to the metabolic assay in Macrophage Serum Free Medium (ThermoFisher, Waltham, MA, USA) and stimulated for $24 \mathrm{~h}$ with $50 \mathrm{ng} / \mathrm{mL}$ IL1 $\beta$ and 20 $\mathrm{ng} / \mathrm{mL}$ IFN $\gamma$ or $50 \mathrm{ng} / \mathrm{mL}$ IL4 (all from R\&D, Minneapolis, MN, USA) to induce an M1 or M2 phenotype, respectively [7]. The efficiency of the polarization was confirmed by measuring the gene expression of typical M1 (iNOS, Tnfa, Il6) and M2 (Arg1, Retnla, Mrc1) markers after the treatment with IL1ß/IFN $\gamma$ and IL4 (Figure S1).

\section{hPSC-Derived Microglia-Like Cells}

Human pluripotent stem cells (hPSC) were differentiated into monocytes and subsequently transdifferentiated into microglia-like cells [38]. Briefly, non-adherent cells were harvested on day 17, 21, 25, 28 and 32, and monocytes were selected by using CD14-labelled magnetic beads (Miltenyi, Bergisch Gladbach, Germany), following the manufacturer's instructions. For transdifferentiation of monocytes to microglia, 500,000 freshly isolated monocytes were plated in one well of a 6-well plate in microglia differentiation medium (Neurobasal medium, N2, B27, lactic acid, sodium pyruvate, glutamax, biotin, ascorbic acid, $\mathrm{NaCl}$, Albumax I and penicillin/streptomycin) containing $10 \mathrm{ng} / \mathrm{mL}$ IL34 and M-CSF. 
Medium was changed every day and the number of adherent microglia per well was between $0.5-1 \times 10^{6}$ cells. After obtaining microglia-like cells, they were replated in 24-well plates at a density of 150,000 cells/well, and stimulated for $24 \mathrm{~h}$ with $100 \mathrm{ng} / \mathrm{mL}$ LPS (Sigma Aldrich, St. Louis, MO, USA). Approval for the use of human embryonic stem cells was obtained from the ethics committee of UZ Leuven (AMV/22042013 and SBB219.2012/1000).

\section{In vivo LPS Challenge}

Twelve-week old control mice were injected intraperitoneally with $100 \mu \mathrm{L}$ LPS ( $1 \mathrm{mg} / \mathrm{kg}$, Sigma Aldrich, St. Louis, MO, USA or sterile saline as previously reported [39,40]. After $4 \mathrm{~h}$, mice were sacrificed and brains were snap frozen in liquid nitrogen and stored at $-80{ }^{\circ} \mathrm{C}$. Tissue was further processed for qRT-PCR as described below.

\section{RNA Isolation and qRT-PCR}

RNA isolation was performed by Trizol extraction for tissue samples, and by using the PureLink RNA Mini Kit for microglia, both according to the manufacturer's protocol (ThermoFisher, Waltham, MA, USA). Subsequently, RNA was converted to cDNA by using the QuantiTect reverse transcription Kit (Qiagen, Hilden, Germany). Quantitative realtime transcription PCR was performed on samples in triplicate with the PowerUp SYBR Green Master Mix (Thermo Fisher, Waltham, MA, USA) using the ABI PRISM 7500 Real Time PCR system (Applied Biosystems, Foster City, CA, USA). Data obtained were quantified using the $2^{-\Delta \Delta C T}$ method [41]. The expression of selected genes was normalized to the expression of the house keeping gene, $\beta$-actin. All primers were purchased from IDT or Eurogentec. The list of primers used can be found in Supplementary Table 1.

\section{Western Blot}

Primary microglia were seeded in a 6-well plate at a density of $1 \times 10^{6}$ cells/well and exposed to IL1 $\beta /$ IFN $\gamma$ or IL4 as described above. After the stimulation, cells were lysed in RIPA buffer (ThermoFisher, Waltham, MA, USA) containing protease inhibitors (Roche, Basel, Switzerland) and collected by scraping. Total protein content was determined using the Pierce ${ }^{\mathrm{TM}}$ BCA Protein Assay Kit (ThermoFisher, Waltham, MA, USA) following the manufacturer's instructions. Subsequently, 10-30 $\mu \mathrm{g}$ of protein was subjected to SDS-polyacrylamide gel electrophoresis (10\% gels). After the separation, proteins were blotted onto a polyvinylidene fluoride membrane and immunostaining was performed. The following primary antibodies were used, ACOX1 (against $51 \mathrm{kDa}$ subunit; 1/5000 [42]), MFP2 (1/400, ProteinTech, Manchester, UK) and vinculin (1/2000, Sigma Aldrich, St. Louis, MO, USA) together with HRP-conjugated secondary antibodies (1/5000, Agilent, Santa Clara, CA, 
USA). Detection was performed using an ECL detection reagent (GE Healthcare Life Science, Marlborough, MA, USA) and the ChemiDoc MP System (BioRad, Hercules, CA, USA). Image Lab software (BioRad, Hercules, CA, USA) was used for image processing and quantification of western blot bands.

\section{Metabolic Assays}

Glycolysis and mitochondrial $\beta$-oxidation were determined by measuring the production of ${ }^{3} \mathrm{H}_{2} \mathrm{O}$ after incubating the cells with labeled glucose or palmitic acid, respectively [43,44]. Briefly, cells were seeded in a 48-well plate at a density of 75,000 cells/well and polarized as described above. For glycolysis, cells were incubated with $0.4 \mu \mathrm{Ci} / \mathrm{mL}\left[5-{ }^{3} \mathrm{H}\right]-\mathrm{D}-$ glucose (Perkin Elmer, Waltham, MA, USA) and for mitochondrial $\beta$ oxidation with $2 \mu \mathrm{Ci} / \mathrm{mL}\left[9,10-{ }^{3} \mathrm{H}\right]$ palmitate (Perkin Elmer, Waltham, MA, USA). After $3 \mathrm{~h}$ of incubation, the medium was transferred into glass vials and sealed with rubber stoppers containing hanging wells with Whatman paper soaked with water. After $48 \mathrm{~h}$ of incubation radioactivity in the paper was determined by liquid scintillation counting.

Glutamine oxidation was determined by measuring production of ${ }^{14} \mathrm{CO}_{2}$ after incubating the cells with labeled glutamine [43,44]. Briefly, cells were seeded in a 12-well plate at a density of 300,000 cells/well and polarized as described previously. Cells were incubated with $0.5 \mu \mathrm{Ci} / \mathrm{mL}$ [U- ${ }^{14}$ C]glutamine (Perkin Elmer, Waltham, MA, USA) for $6 \mathrm{~h}$ after which $250 \mu \mathrm{L}$ of $12 \%$ perchloric acid was added to stop the reaction and release ${ }^{14} \mathrm{CO}_{2}$ from the medium, which was captured on Hyamine soaked filter papers overnight. The radioactivity was measured as mentioned above.

Glucose oxidation was determined in the same manner as glutamine oxidation $[43,44]$. Briefly, cells where incubated with $0.55 \mu \mathrm{Ci} / \mathrm{mL}\left[1-{ }^{14} \mathrm{C}\right]-$ D-glucose (Perkin Elmer) or [6- $\left.{ }^{14} \mathrm{C}\right]$-D-glucose (Perkin Elmer, Waltham, MA, USA) and the production of ${ }^{14} \mathrm{CO}_{2}$ was determined by liquid scintillation counting. Oxidation of $1{ }^{14} \mathrm{C}$-glucose represents oxidation through both the pentose phosphate pathway (PPP) and tricarboxylic acid (TCA) cycle while oxidation of $6-{ }^{14} \mathrm{C}$ glucose represents oxidation through the TCA cycle alone. The activity of the PPP was calculated as the difference between $1{ }^{14} \mathrm{C}$ and $6-{ }^{14} \mathrm{C}$ glucose oxidation $[44,45]$.

Peroxisomal $\beta$-oxidation was determined similar to glutamine and glucose oxidation. Briefly, cells were incubated with $0.03 \mu \mathrm{Ci} / \mathrm{mL} 1-{ }^{14} \mathrm{C}$ labeled 2-methylhexadecanoic acid (synthetic BCFA) (5 $\mu \mathrm{M})$ [46] bound to defatted albumin (molar ratio 2) for $24 \mathrm{~h}$, followed by ${ }^{14} \mathrm{CO}_{2}$ collection and liquid scintillation counting [47].

Fatty acid synthesis was measured by determining the incorporation of $\left[1,2-{ }^{14} \mathrm{C}\right]$-acetate into triacylglycerides (TAG) as described [48]. Briefly, cells were seeded in a 6-well plate at a density of $1 \times 10^{6}$ cells/well and polarized as described above. Cells were incubated with $0.5 \mu \mathrm{Ci} / \mathrm{mL}[1,2-$ ${ }^{14} \mathrm{C}$-acetate (Perkin Elmer, Waltham, MA, USA for $24 \mathrm{~h}$ after which they were washed with phosphate-buffered saline and collected by scraping. 
Lipids were extracted by methanol/chloroform extraction as described [49] and spotted together with appropriate standards onto silica gel plates. The plates were developed in hexane/diethylether/acetic acid $(70 / 30 / 1, v / v / v)$ and lipids were visualized with iodine vapor. The amount of radioactive label incorporated in the TAG was quantified by scraping the silica gel of the corresponding area and scintillation counting.

All measurements were normalized to cell number or phospholipid content, which was determined as described [50]. Data were corrected for the total concentration of the substrate (non-labeled and radiolabeled) present in the medium at the beginning of the incubation with the radiolabeled substrate.

\section{Statistical Analysis}

For statistical analysis, GraphPad Prism software (version 7.0) was used and the following tests were performed: unpaired two-sided Student's $t$-test or one-way ANOVA repeated measures followed by Tukey post-test. Data are shown as mean \pm SEM and statistical significance was set at $p<0.05$.

\section{RESULTS}

\section{Glucose Metabolism Is Increased in M1 Microglia}

Primary microglial cultures from newborn mice were polarized into a pro-inflammatory M1 and into an anti-inflammatory M2 state, by exposing them to IL1 $\beta /$ IFN $\gamma$ and IL4, respectively [7]. In view of the wellknown activation of glycolysis in pro-inflammatory macrophages, we first determined the activity of this pathway by estimating the gene expression of several key glycolytic enzymes (Pfkfb3, Pfkfb1 and Hk3) and we measured the rate of ${ }^{3} \mathrm{H}_{2} \mathrm{O}$ production after incubating the cells with radiolabeled glucose. Gene expression analysis revealed an upregulation of both $P f k f b 3$ and $H k 3$ and downregulation of $P f k f b 1$ in M1 microglia, which did not reach statistical significance (Figure 1A-C). However, the ratio $P f k f b 3 / P f k f b 1$ was significantly higher, indicative of increased glycolysis in pro-inflammatory microglia (Figure 1D). Indeed, both $P f k f b 1$ and Pfkfb3 encode 6-phosphofructo-2-kinase/fructose-2,6-biphosphatase, however, $P f k f b 3$ encodes the enzyme with higher catalytic activity of the kinase versus the phosphatase leading to higher concentrations of fructose-2,6-biphosphate, an allosteric activator of phosphofructokinase (PFK)1 (Figure 1F). Consequently, activation of PFK1, the enzyme catalyzing the third step of glycolysis, enhances glycolysis even if enough ATP is available [51]. This was confirmed by measuring pathway activity using radiolabeled glucose, which showed a 2-fold increase in glycolytic flux in M1 microglia (Figure 1E). These data are in line with other studies performed in BV-2 and primary microglia [23,25]. Moreover, similar data were obtained for microglia stimulated with another M1 stimulus, LPS (data not shown). Strikingly, in contrast to M2 macrophages [52], we observed no changes regarding glycolysis in M2 microglia (Figure 1). 

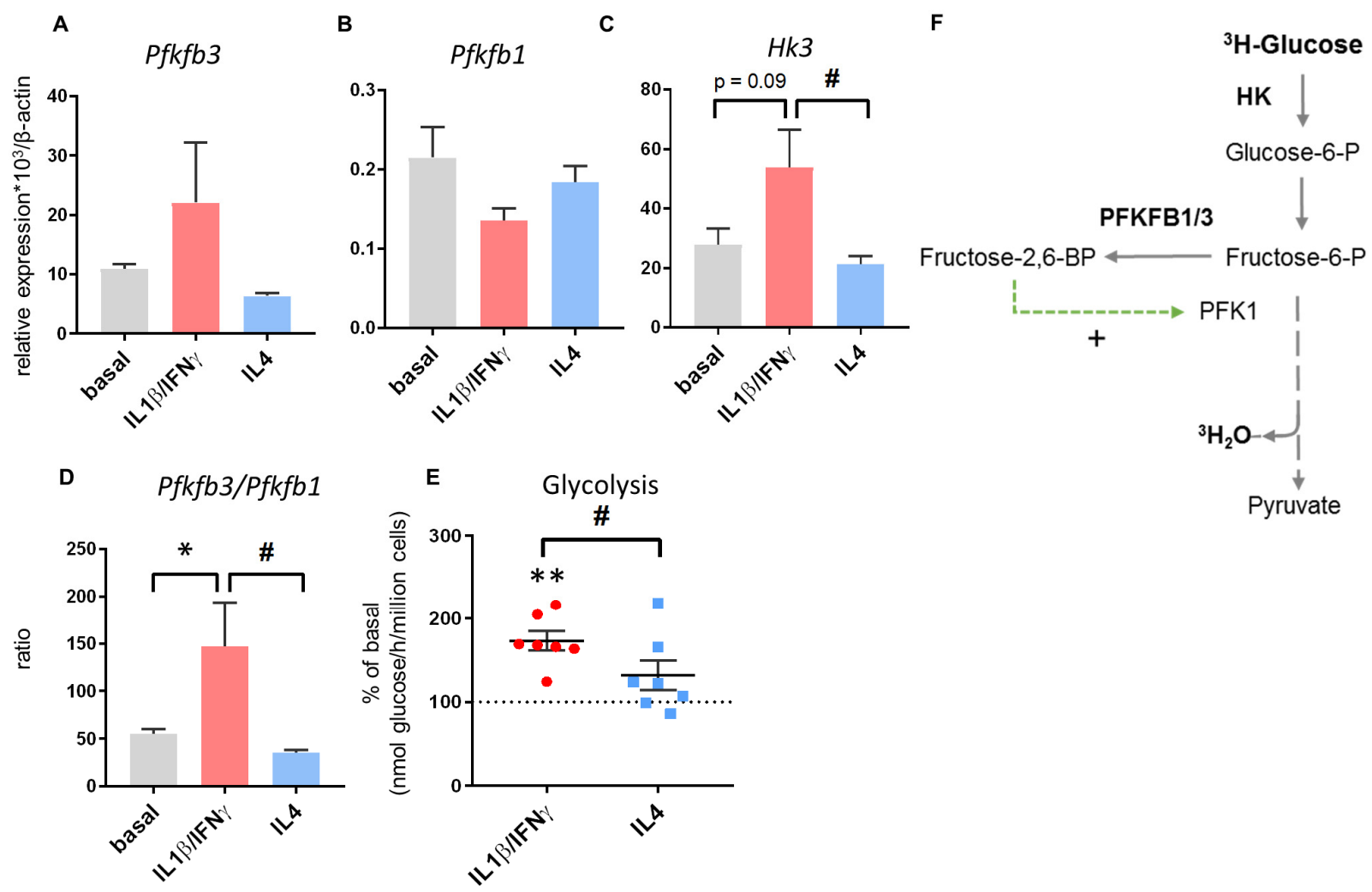

Pyruvate

Figure 1. Increased glycolysis in pro-inflammatory microglia. (A-D) Gene expression of glycolytic enzymes $P f k f b 3$ (A), $P f k f b 1$ (B) and $H k 3$ (C) and the ratio of $P f k f b 3 / P f k f b 1$ (D) in pro- and anti-inflammatory microglia ( $n=7-9$ /group); (E) Glycolytic activity in pro- and anti-inflammatory microglia compared to basal conditions expressed as \% of basal activity (1230 nmol glucose/h/million cells) ( $n$ = 7/group); (F) Simplified scheme of glycolysis; green arrow represents positive regulation. Bars represent mean \pm SEM. Note: Red color represents pro-inflammatory M1 state while blue color represents anti-inflammatory M2 state. Statistical differences based on One-way ANOVA test: ${ }^{*} p<0.05,{ }^{* *} p<0.01$ (pro- or anti-inflammatory state compared to the basal state), $\# p<0.05$ (pro-inflammatory state versus anti-inflammatory state).

The end product of glycolysis is pyruvate, which can give rise to either lactate or to acetyl-CoA. The latter subsequently enters the tricarboxylic acid (TCA) cycle generating energy laden NADH and FADH2, which can subsequently fuel the electron transport chain (Figure 2E). Interestingly, in contrast to BV-2 microglia, which show increased lactate dehydrogenase (LDH) activity and lactate production [24], we did not observe any change in Ldha expression in primary M1 microglia (Figure 2A). We therefore hypothesized that the increased glycolysis in M1 microglia might provide pyruvate for acetyl-CoA synthesis, fueling the TCA cycle and enabling full glucose oxidation. To test this, we estimated gene expression of Pdha1, crucial for pyruvate to acetyl-CoA conversion, in M1 microglia. The expression of Pdha1 was unaltered in M1 microglia (Figure 2B). However, gene expression of $P d k 1$, which regulates pyruvate dehydrogenase (PDH) activity was downregulated (Figure 2C). Notably, PDK1 phosphorylates PDH, which leads to its inactivation, subsequently blocking conversion of pyruvate into acetyl-CoA and its entrance into the 
TCA cycle (Figure 2E) [53]. Thus, as we observed decreased Pdk1 expression in M1 microglia (Figure 2C), we speculated that these cells have higher rates of pyruvate to acetyl-CoA conversion. To examine this, we assessed $6-{ }^{14} \mathrm{C}$ glucose oxidation, which is a measure of glucose oxidation through the TCA cycle [54]. We observed higher rates of $6{ }^{14} \mathrm{C}$-glucose oxidation in M1 microglia (Figure 2D), proving that M1 microglia increase glucose utilization not only through glycolysis, but also through the TCA cycle. Additionally, we examined whether M2 microglia exhibit any alterations, but we observed no changes in the gene expression of Ldha, Pdha1 and Pdk1, nor in glucose oxidation through the TCA cycle (Figure 2).
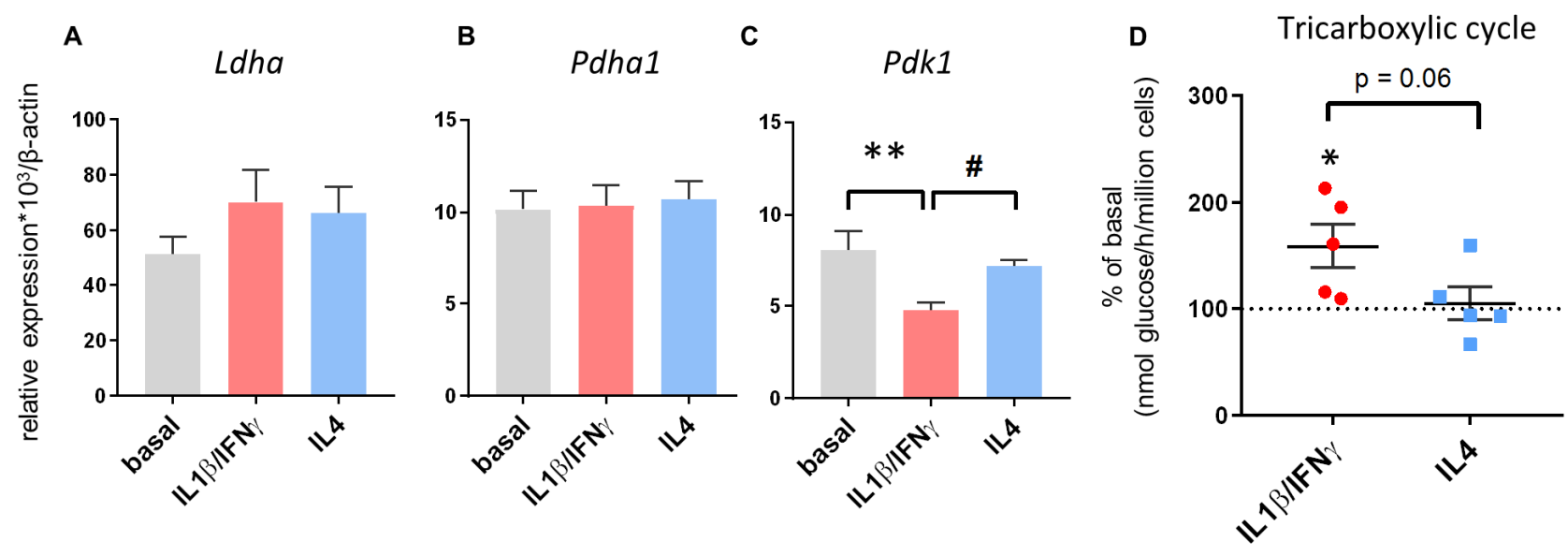

E

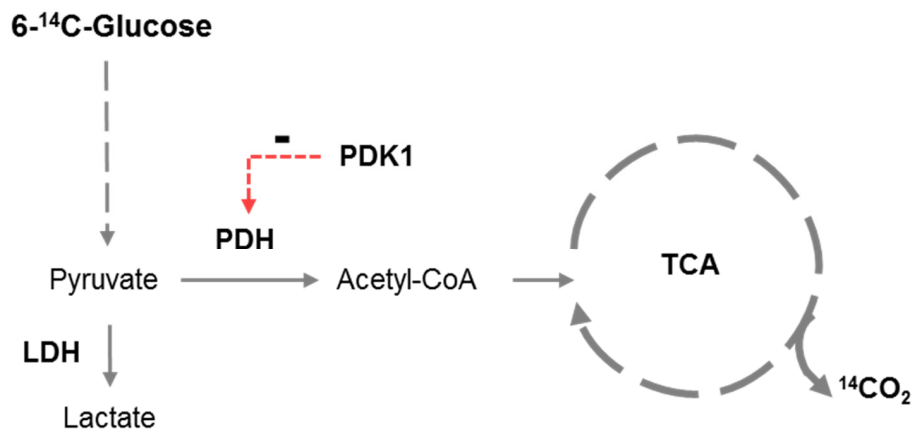

Figure 2. Increased glucose oxidation through the TCA cycle in pro-inflammatory microglia. (A-C) Gene expression of enzymes Ldha (A), Pdha1 (B) and Pdk1 (C) in pro- and anti-inflammatory microglia $(n=$ 7-9/group); (D) Glucose oxidation through the TCA cycle in pro- and anti-inflammatory microglia compared to basal conditions expressed as the \% of basal activity ( $0.68 \mathrm{nmol}$ glucose $/ \mathrm{h} / \mathrm{million}$ cells) $(n=$ 5/group); (E) Simplified scheme of glucose fate after the production of pyruvate through glycolysis. Note: red arrow represents negative regulation. Bars represent mean \pm SEM. Red color represents proinflammatory M1 state while blue color represents anti-inflammatory M2 state. Statistical differences based on One-way ANOVA test: ${ }^{*} p<0.05,{ }^{* *} p<0.01$ (pro- or anti-inflammatory state compared to the basal state) $\# p<0.05$ (pro-inflammatory state versus anti-inflammatory state).

Glucose can also be metabolized via the pentose phosphate pathway (PPP), in which NADPH and pentoses are generated. NADPH fuels the activities of NADPH oxidase and iNOS, which produce reactive oxygen species (ROS) and nitric oxide (NO), respectively, while pentoses serve 
as building blocks for nucleotide synthesis. Interestingly, a study performed in BV-2 microglia indicated increased PPP in the M1 state based on a higher glucose-6-phosphate dehydrogenase (G6PD) activity [24]. Here, we first determined the gene expression of G6pd as well as the expression of the PPP regulator, sedoheptulokinase (Shpk). Particularly, Shpk regulates the PPP through the production of seduheptulose-7-phosphate, and its downregulation leads to the stimulation of PPP and vice versa (Figure 3D) [55]. We observed a slight decrease in G6pd expression in primary M1 microglia $(p=0.06)$ and no significant changes in Shpk expression (Figure $3 \mathrm{~A}, \mathrm{~B})$. At the level of pathway activity in M1 microglia, there was a trend for an increased PPP, but this did not reach statistical significance (Figure 3C). Additionally, we examined whether M2 microglia exhibit any alterations, but we observed no changes in the gene expression of G6pd and Shpk, nor in the PPP activity (Figure 3).

Altogether our data demonstrate increased glucose turnover in proinflammatory microglia with most pronounced effects on glycolysis.

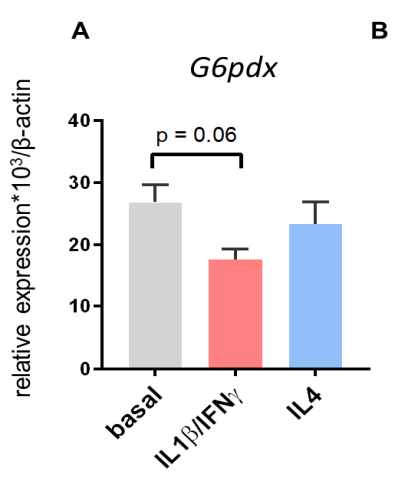

B

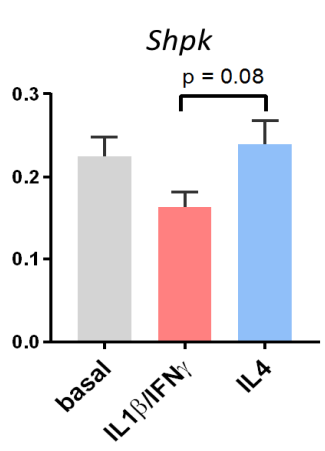

C Pentose phosphate pathway

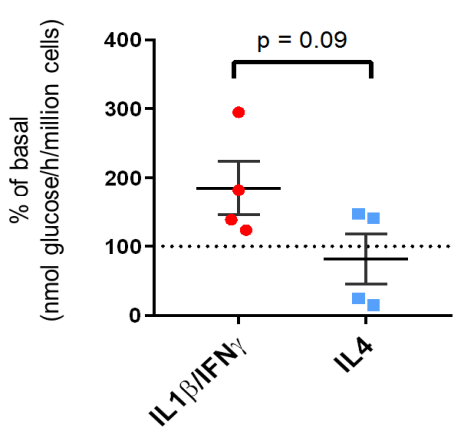

D

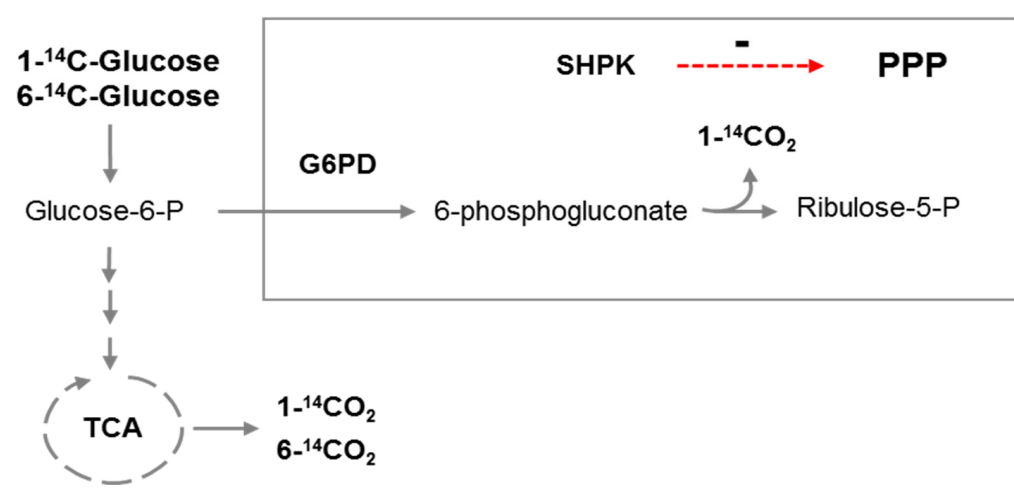

Figure 3. No significant changes in the PPP during microglia polarization. (A-B) Gene expression of G6pd (A) and Shpk (B) in pro- and anti-inflammatory microglia ( $n=7-9$ /group); (C) Glucose oxidation through the PPP (calculated as difference between $1-{ }^{14} \mathrm{C}$ and $6-{ }^{14} \mathrm{C}$ glucose oxidation) in pro- and anti-inflammatory microglia compared to basal conditions expressed as the $\%$ of basal activity ( $0.53 \mathrm{nmol}$ glucose/h/million cells) ( $n=4 /$ group); (D) Schematic representation of glucose usage through PPP. Note: red arrow represents negative regulation. Bars represent mean \pm SEM. Red color represents pro-inflammatory M1 state while blue color represents antiinflammatory M2 state. Statistical differences based on One-way ANOVA test. 


\section{Increased Glutamine Oxidation in M1 Microglia}

Next to glucose oxidation, we also investigated glutamine oxidation in differentially activated microglia. More precisely, we measured oxidation of radiolabeled glutamine together with estimating the gene expression of Glud1 and Got1, involved in the entrance of glutamine into the TCA cycle. Glud1 and Got1, encode glutamate dehydrogenase (GLUD) and glutamateoxaloacetate transaminase (GOT), respectively, both catalyzing the conversion of glutamate to a-ketoglutarate (aKG) (Figure 4D). In M1 microglia, we observed a reduction in Glud1 expression, while the expression of Got1 was increased (Figure 4A,B). Since these enzymes catalyze the same reaction, and as we saw their opposite regulation in M1 microglia, we estimated glutamine oxidation by using radiolabeled glutamine. The data demonstrated increased glutamine oxidation in M1 microglia (Figure 4C), supported by increased Got1 expression. In contrast to M1 microglia, no evident adaptations were observed in M2 microglia (Figure 4).

A

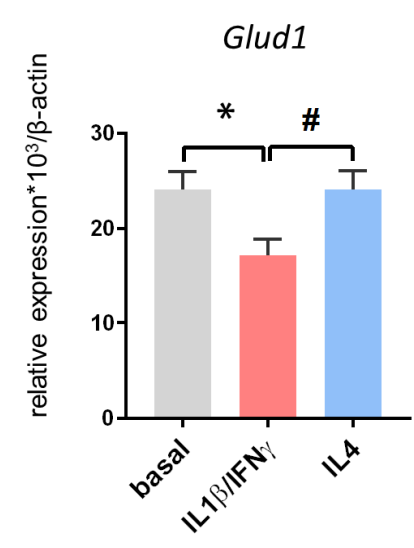

B

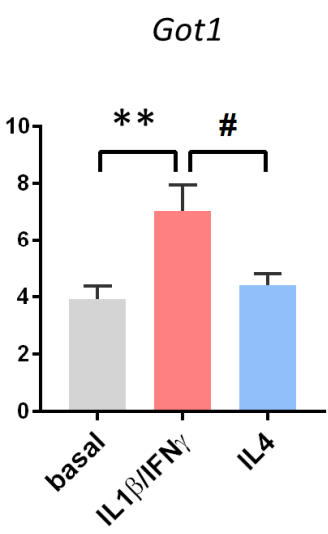

C Glutamine oxidation

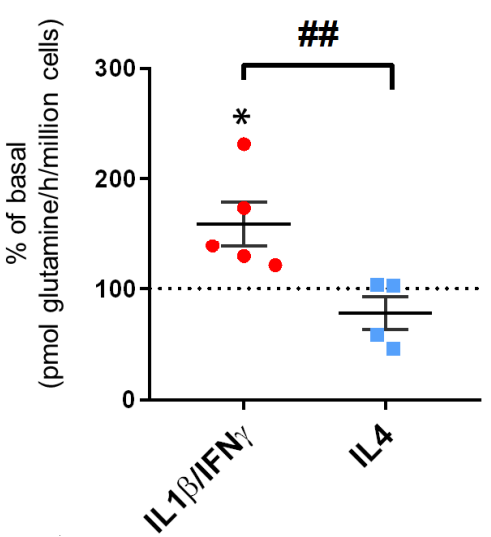

D

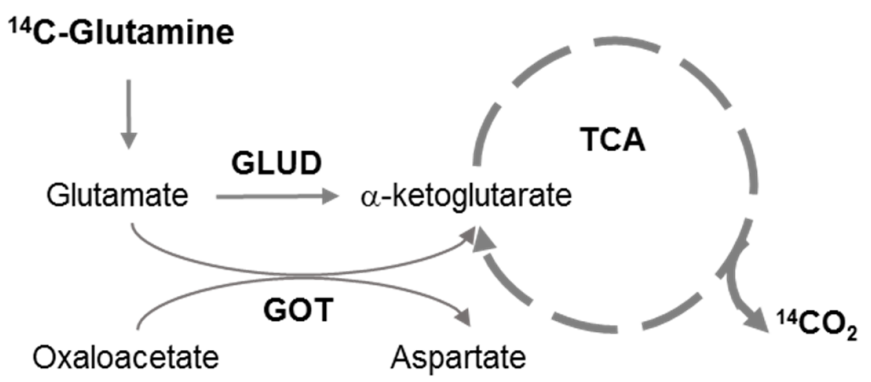

Figure 4. Glutamine oxidation is increased in pro-inflammatory microglia. (A-B) Gene expression of Glud1 (A) and Got1 (B) in pro- and anti-inflammatory microglia ( $n=8-9 /$ group); (C) Glutamine oxidation in pro- and anti-inflammatory microglia compared to basal conditions expressed as the $\%$ of basal activity (0.36 pmol glutamine/h/million cells) ( $n=4-5 /$ group); (D) Schematic representation of glutamine entrance into the TCA cycle. Note: Bars represent mean \pm SEM. Red color represents pro-inflammatory M1 state while blue color represents anti-inflammatory M2 state. Statistical differences based on One-way ANOVA test: ${ }^{*} p<0.05,{ }^{* *} p<0.01$ (pro- or anti-inflammatory state compared to the basal state) $\# p<0.05, \# \# p<0.01$ (pro-inflammatory state versus anti-inflammatory state). 


\section{Fatty Acid Oxidation and Fatty acid Synthesis Are Oppositely Regulated in M1 and M2 Microglia}

In order to get insight into fatty acid metabolism in differentially activated microglia, we investigated both fatty acid degradation and fatty acid synthesis. Thereby, we did not only focus on the degradation of long and medium chain fatty acids via mitochondrial $\beta$-oxidation, but also assessed peroxisomal $\beta$-oxidation, which is essential for the degradation of specific fatty acids such as very long chain fatty acids (VLCFA) and branched chain fatty acids (BCFA) as well as the synthesis and degradation of polyunsaturated fatty acids (PUFA) [56,57].
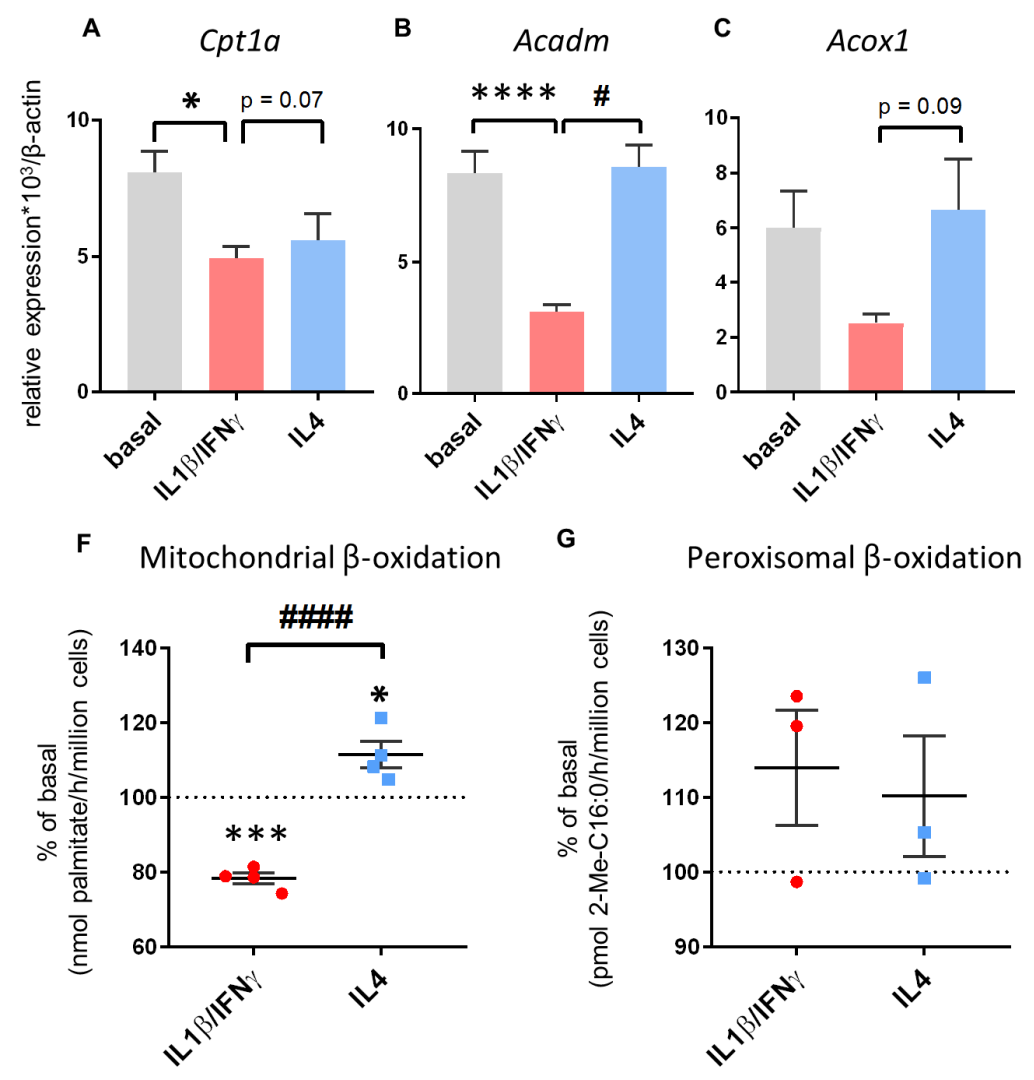

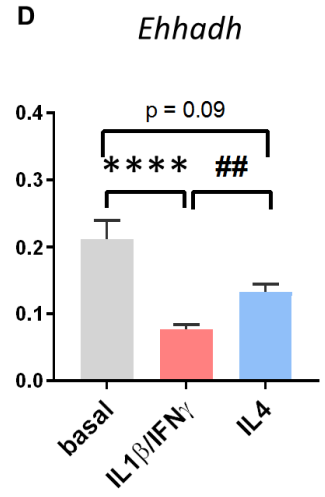

E $\quad H s d 17 b 4$
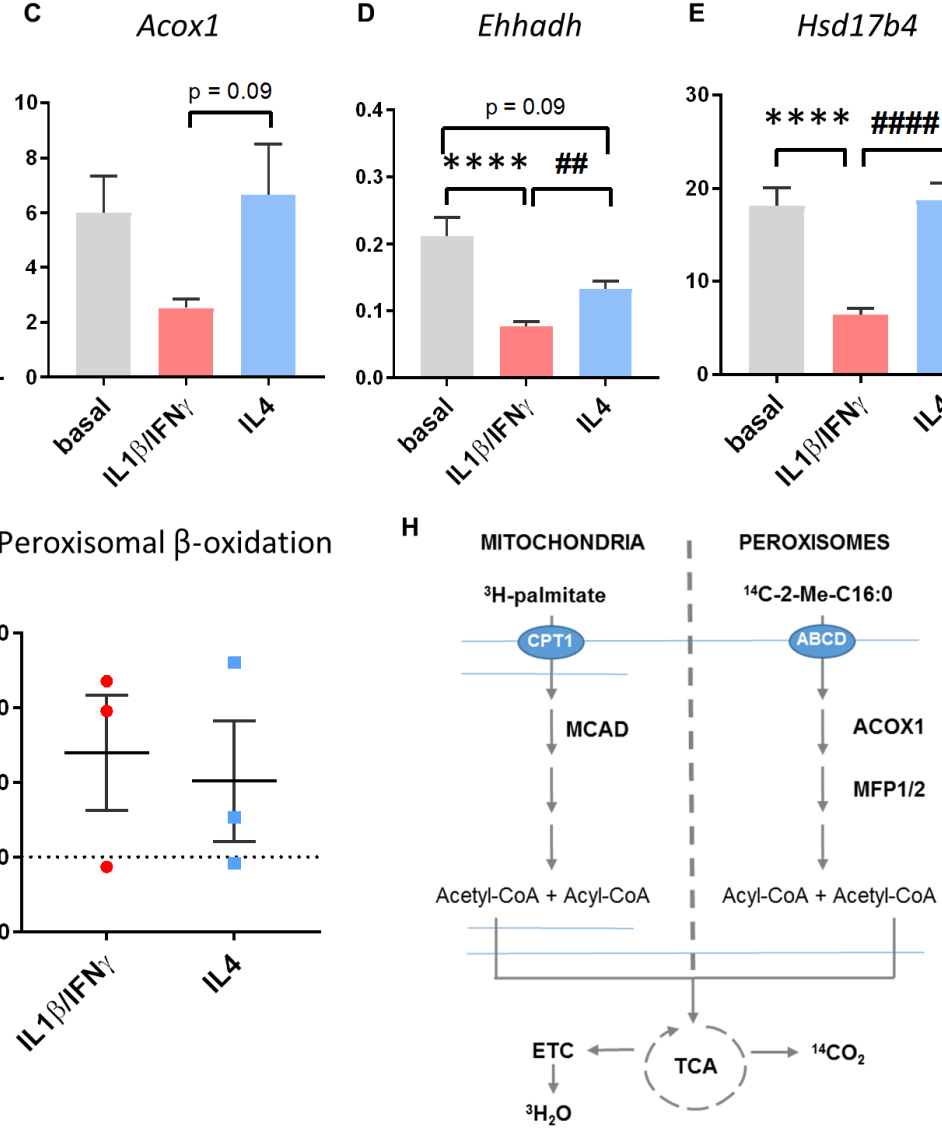

Figure 5. Regulation of fatty acid oxidation in polarized microglia. (A-E) Gene expression of enzymes involved in mitochondrial (A-B) and peroxisomal $\beta$-oxidation (C-E) in pro- and anti-inflammatory microglia ( $n=8-9 /$ group); (F-G) Mitochondrial (F) and peroxisomal (G) $\beta$-oxidation in pro- and antiinflammatory microglia compared to basal conditions expressed as the \% of basal activity (47 nmol palmitate/h/million cells and 24 pmol 2-Me-C16:0/h/million cells) ( $n=3-4 /$ group) (H) Schematic representation of mitochondrial and peroxisomal $\beta$-oxidation; ETC-electron transport chain. Note: Bars represent mean \pm SEM. Red color represents pro-inflammatory M1 state while blue color represents antiinflammatory M2 state. Statistical differences based on One-way ANOVA test: ${ }^{*} p<0.05,{ }^{* * *} p<0.001,{ }^{* * * *} p<$

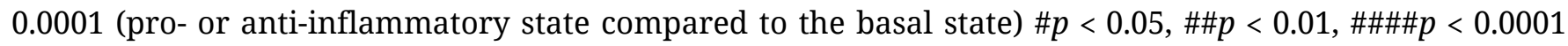
(pro-inflammatory state versus anti-inflammatory state).

First, we estimated the gene expression of enzymes involved in both mitochondrial (Cpt1a and Acadm (encoding MCAD) and peroxisomal 
ß-oxidation (Acox1, Ehhadh (encoding MFP1) and Hsd17b4 (encoding MFP2) which revealed strong and robust suppression of both mitochondrial and peroxisomal $\beta$-oxidation transcripts in M1 microglia (Figure 5A-E). On the other hand, no changes were observed in M2 microglia with the exception of Ehhadh, which was downregulated (Figure 5D).

To further strengthen the gene expression data, we measured the activity of both mitochondrial and peroxisomal $\beta$-oxidation by using radiolabeled palmitate and 2- methylhexadecanoic acid (2-Me-C16:0) [46], respectively (Figure 5H). In line with the gene expression data, we observed suppression of palmitate oxidation in M1 microglia (Figure 5F). In contrast, M2 microglia showed a slight increase in mitochondrial $\beta$-oxidation (Figure 5F). Surprisingly, even though we observed a strong and consistent suppression of peroxisomal $\beta$-oxidation enzymes in M1 microglia at the level of gene expression, the breakdown of 2-methylhexadecanoic acid was similar in all microglial states (Figure 5G).

To further investigate this discrepancy, we also determined the protein levels of the peroxisomal enzymes, ACOX1 and MFP2, in polarized microglia. Interestingly, we observed no changes in these proteins based on western blot analysis. This explains the unchanged pathway activity despite the strong suppression of enzymes at the level of gene expression (Figure 6).

A

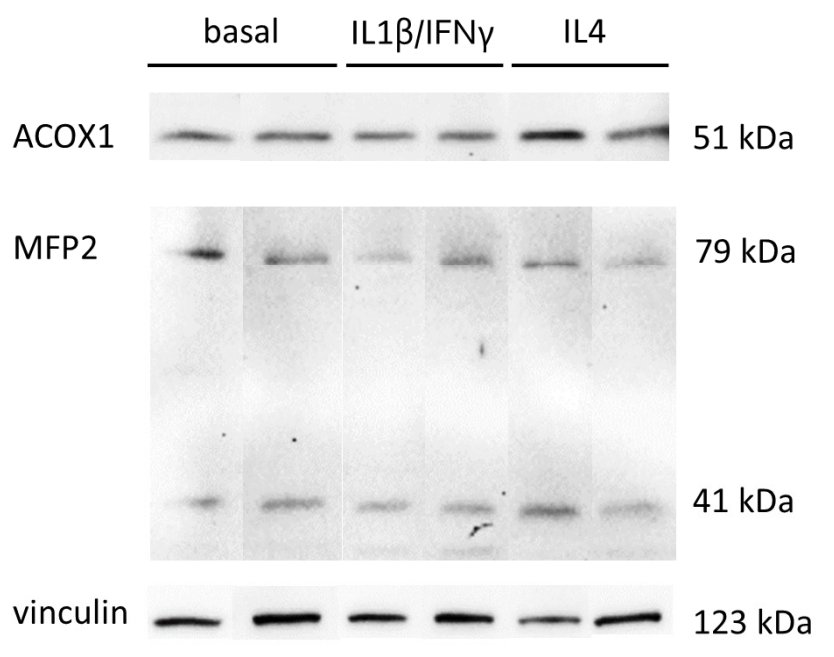

B ACOX1
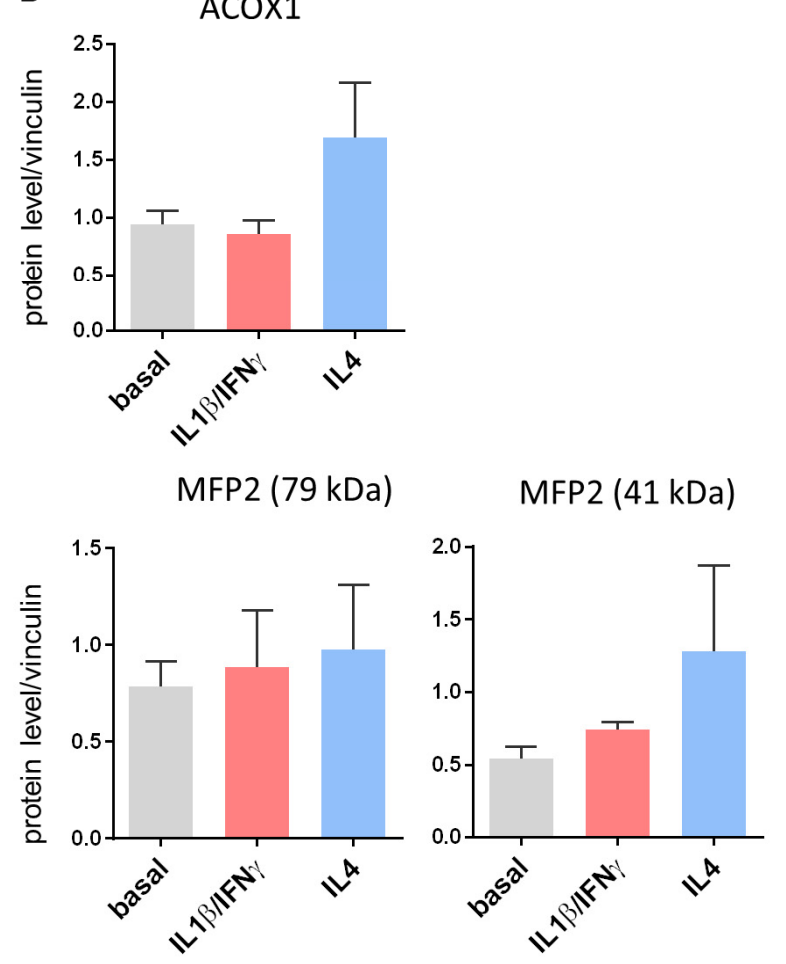

Figure 6. Unchanged protein levels of peroxisomal enzymes in polarized microglia. (A,B) Western blot analysis of ACOX1 and MFP2 protein levels in pro- and anti-inflammatory microglia compared to basal conditions ( $n=2$ /group). A representative blot (A) and quantification (B) are shown. Note: Bars represent mean \pm SEM. Red color represents pro-inflammatory M1 state while blue color represents antiinflammatory M2 state. 
Beside the fatty acid degradation, we were also interested whether microglia show differences in fatty acid synthesis during polarization. We measured both the gene expression of fatty acid synthase (Fasn) as well as incorporation of radiolabeled acetate into triacylglycerides (TAG), a measure of fatty acid synthesis. Gene expression analysis showed a decreased Fasn expression, accompanied by a decrease in the pathway activity in M1 microglia (Figure 7A,B). In contrast, in M2 microglia, we observed a strong increase in the pathway activity, even though no changes were observed in the expression of Fasn (Figure 7A,B).

Altogether our data demonstrate opposite regulation of FAO and fatty acid synthesis in M1 and M2 microglia, as M1 microglia suppress these pathways, while M2 microglia boost them. The exception was peroxisomal FAO, which was unaltered between the microglia states.

A

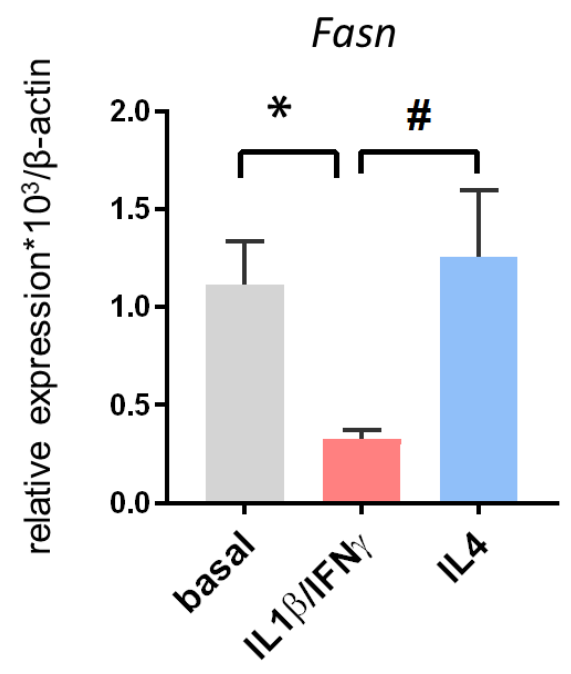

B

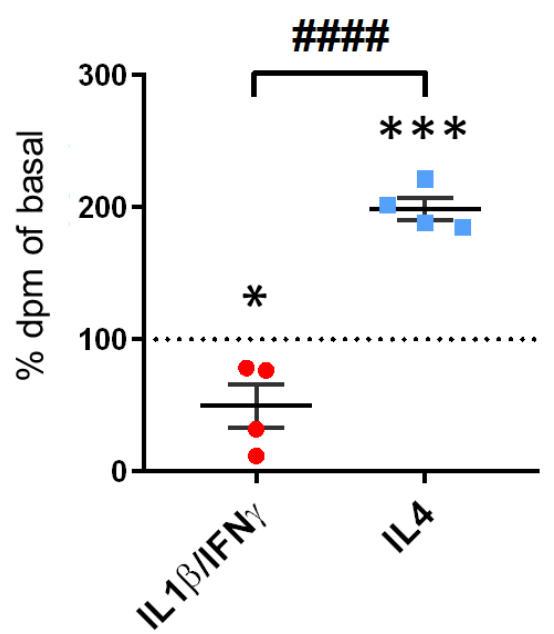

Figure 7. Fatty acid synthesis is suppressed in pro-inflammatory microglia and boosted in antiinflammatory microglia. (A) Gene expression of Fasn enzyme involved in fatty acid synthesis in pro- and anti-inflammatory microglia ( $n=9$ /group); (B) Fatty acid synthesis in pro- and anti-inflammatory microglia compared to basal conditions expressed as the $\%$ of basal activity ( $77 \mathrm{dpm} / 24 \mathrm{~h} /$ million cells) ( $n=4$ /group). Note: Bars represent mean \pm SEM. Red color represents pro-inflammatory M1 state while blue color represents anti-inflammatory M2 state. Statistical differences based on One-way ANOVA test: ${ }^{*} p<0.05$,

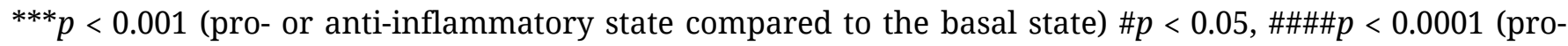
inflammatory state versus anti-inflammatory state).

\section{Human Microglia-Like Cells Also Exhibit Increased Glycolysis in the M1 State}

We clearly demonstrated that the most pronounced metabolic adaptation in pro-inflammatory microglia is the increase in glycolysis. Interestingly, several studies in rodents highlighted beneficial effects of suppressing glycolysis on the outcome of events such as stroke [31]. In order to potentially translate this to patients, an outstanding question is whether pro-inflammatory human microglia display similar metabolic adaptations. To try to answer this question we employed microglia-like 

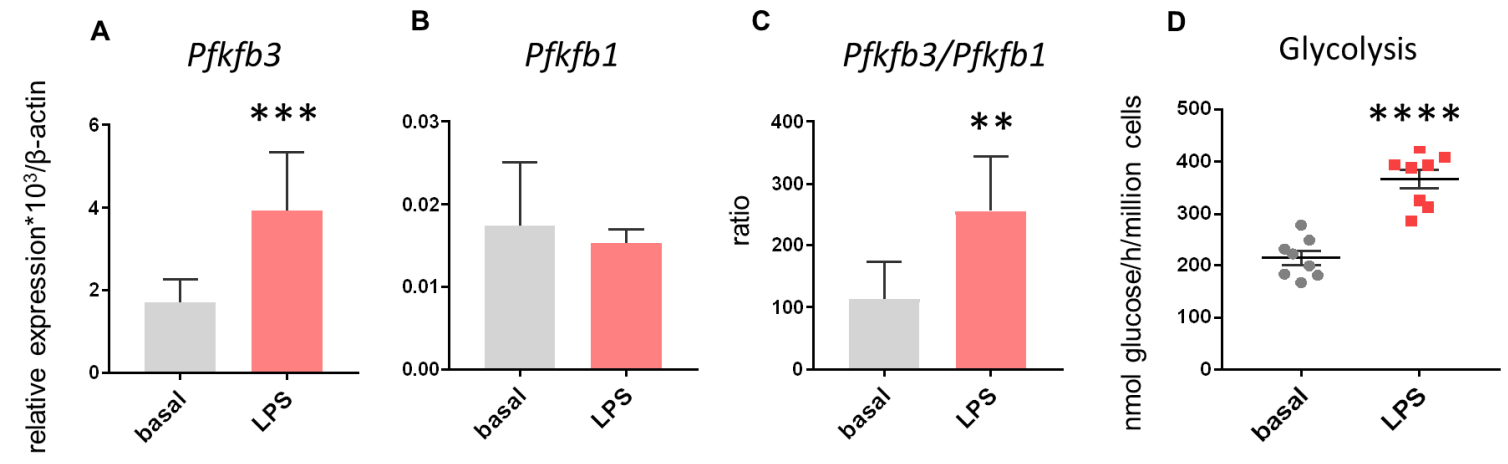

Figure 8. Increased glycolysis in LPS treated hPSC-derived microglia. (A-C) Gene expression of glycolytic enzymes Pfkfb3 (A) and Pfkfb1 (B) and the ratio of Pfkfb3/Pfkfb1 (C) in LPS-treated hPSC-derived microglia-like cells compared to basal conditions ( $n=6$ /group); (D) Glycolytic activity in LPS-treated hPSCderived microglia-like cells compared to basal conditions ( $n=8$ /group). Note: Bars represent mean \pm SEM. Statistical differences based on unpaired $t$-test: ${ }^{* *} p<0.01,{ }^{* * *} p<0.001,{ }^{* * *} p<0.0001$.

\section{Indication of Increased Glycolysis in the Brain after Systemic LPS Administration}

Lastly, in order to assess whether a similar trend of increased glycolysis in pro-inflammatory microglia could be recapitulated in an in vivo setting, we administered LPS intraperitoneally to control mice and estimated gene expression of glycolytic enzymes in the brain. As expected, LPS treatment led to increased expression of pro-inflammatory markers in the brain 4 hours post-injection (Figure 9A-D). Interestingly, this was accompanied by increased expression of the glycolytic enzyme, Pfkfb3, but not $H k 3$ (Figure 9E,G). The gene expression of $P f k f b 1$ was unaltered (Figure 9F), but the ratio Pfkfb3/Pfkfb1 was elevated in LPS-treated mice (Figure 9H). Furthermore, it is likely that microglia are the main cells responsible for the observed changes in the LPS-treated group. It has indeed been shown that microglia, not astrocytes, exhibit activated cytokine expression 4 hours postinjection [58]. These results suggest that pro-inflammatory microglia upregulate glycolytic activity, not only in vitro, but also in vivo, and that this might be mediated through increased $P f k f b 3$ expression. 


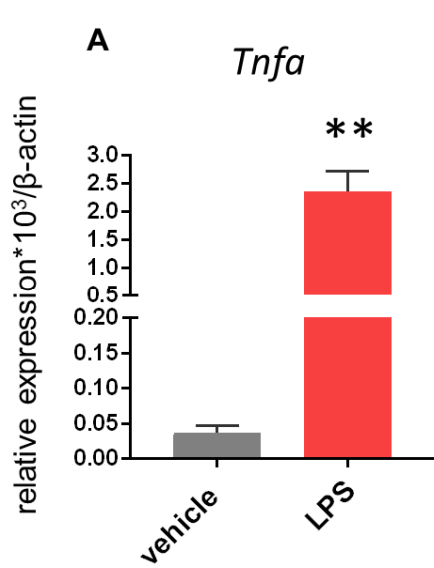

E

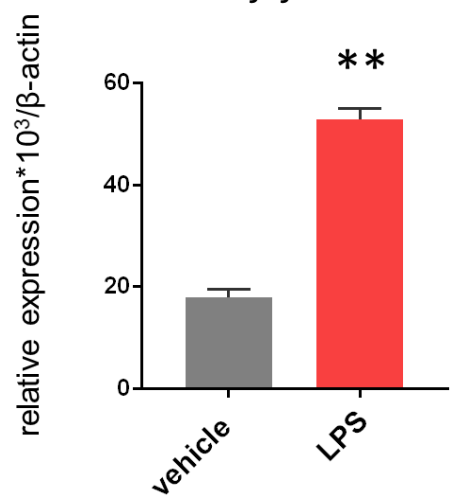

B

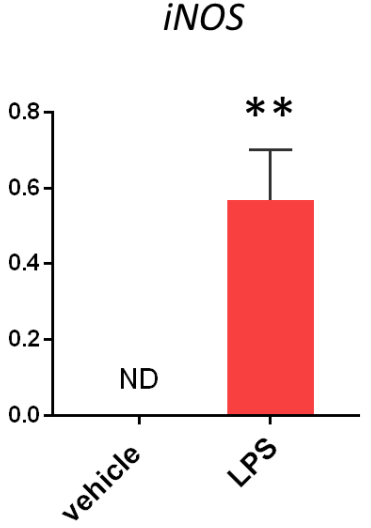

$\mathbf{F}$

Pfkfb1

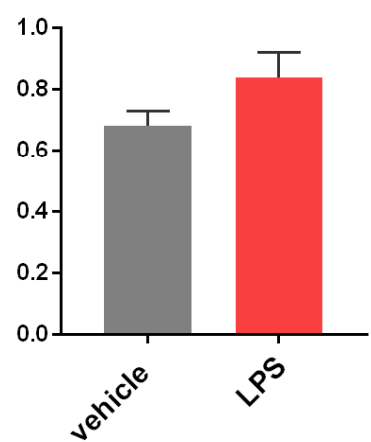

C

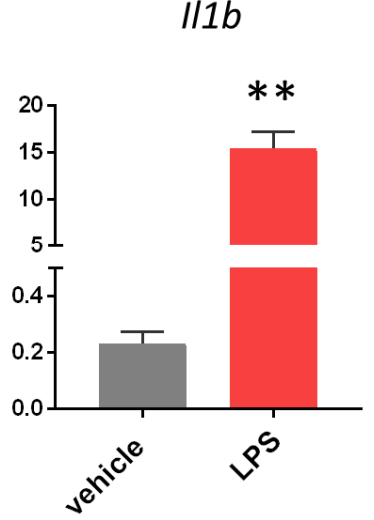

G

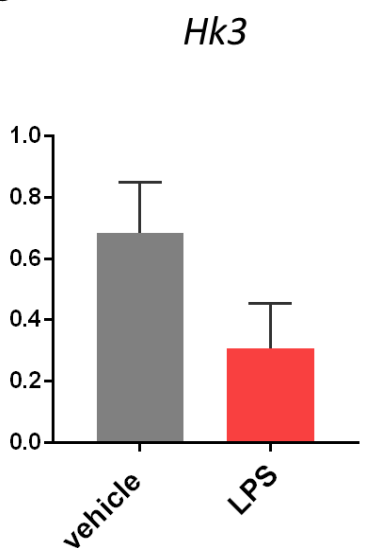

D

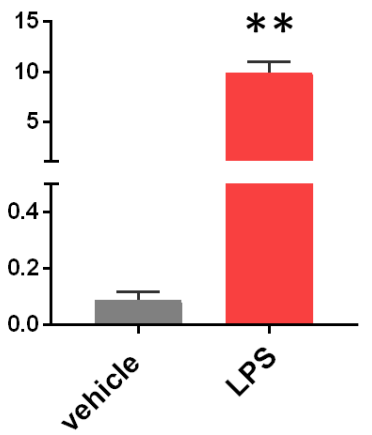

H

$P f k f b 3 / P f k f b 1$

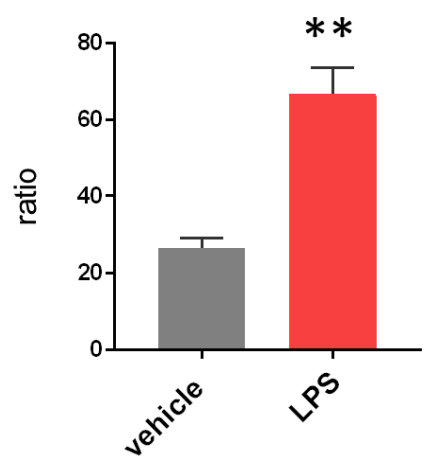

Figure 9. Increased expression of the glycolytic enzyme Pfkfb3 in the brain of LPS-treated mice. (A-H) Gene expression of pro-inflammatory markers (A-D) and glycolytic enzymes (E-H) in the brain of control mice treated with LPS ( $n=4-10$ /group). Note: Bars represent mean \pm SEM. Statistical differences based on unpaired $t$-test: ${ }^{* *} p<$ 0.01 .

\section{DISCUSSION}

In this study, we report that, similar to macrophages, microglia undergo metabolic adaptations during polarization (all data are summarized in Figure 10). We provide a thorough insight into microglia metabolism and point out that glycolysis is a major hallmark of pro-inflammatory microglia. In addition, we revealed FAO and fatty acid synthesis as key features of antiinflammatory microglia.

The increased flux through the glycolytic pathway in primary murine pro-inflammatory, M1 microglia is in line with previous studies using cell lines and indirect approaches. Few studies performed on the BV-2 microglia cell line report an increase in glycolysis in LPS-stimulated microglia [22,23]. This was concluded mainly based on the increase in acidification rate, measured by the XF24 extracellular flux analyzer. Furthermore, a recent study on primary microglia showed increased glycolysis in IFN $\gamma$-stimulated cells, which was accompanied with an increased $P f k f b 3 / P f k f b 1$ ratio [25]. Additionally, in our study, for the first time, we demonstrated that not only murine, but also human microglia- 
like cells exhibit increased glycolysis in a pro-inflammatory setting, implying glycolysis as an M1 microglia feature. Importantly, several studies have already demonstrated that inhibition of glycolysis in microglia can prevent pro-inflammatory microglia activation both in vitro and in vivo, further supporting this notion [31,59,60]. For example, treatment of microglia with 2-deoxyglucose, a glycolytic inhibitor, is able to diminish the NO production in LPS-treated microglia, which could be useful for inhibiting neurotoxic microglia properties [59]. Moreover, several studies have reported beneficial effects of 2-deoxyglucose in Alzheimer's disease (AD) [34], Parkinson's disease [35], epilepsy [36] and ischemic stroke [31]. However, all these studies involved systemic, nonmicroglia specific treatments, so the effect of such approaches could not be assigned directly or solely to microglia. Thus, further research is necessary to determine whether selective targeting of glycolysis in microglia would affect neurodegenerative processes. Interestingly, increased glycolysis has been observed in microglia isolated from mouse models of AD [25,30], highlighting glycolysis as plausible pathway to shape microglia in AD. Based on our data, we propose PFKFB3 as a target to inhibit glycolysis in microglia, as we observed its increased expression in pro-inflammatory settings both in vitro and in vivo. Of note, the PFKFB3 inhibitor, 3PO, was already shown to inhibit glycolysis in several cell types, including macrophages [61,62]. Moreover, as PFKFB3 only drives exaggerated glycolysis [51], we speculate that PFKFB3 inhibition might be a good strategy to inhibit glycolysis selectively in M1 microglia. In particular, it was shown that in macrophages silencing PFKFB3 or 3PO treatment does not affect glycolysis in the resting state, but only in the pro-inflammatory state [62]. However, it will be of importance to further define if inhibition of PFKFB3 reduces glycolysis in microglia and if such approach affects microglia polarization.

In parallel to increased glycolysis in LPS-stimulated BV-2 and primary microglia, few studies report a suppression of OXPHOS [22,23]. Interestingly, this observation was not recapitulated in IFN $\gamma$-stimulated primary microglia [25]. Furthermore, this was concluded based on the oxygen consumption rate, which does not specify the pathway of the oxygen utilization. Indeed, several metabolic reactions will require oxygen, such as glucose oxidation (through the PPP or TCA cycle) as well as glutamine and FAO. In our study, we made a distinction between these pathways and measured fluxes through each individual pathway. We showed here that M1 microglia exhibit increased glucose and glutamine oxidation, while suppressing mitochondrial FAO. On the other hand, M2 microglia showed increased mitochondrial FAO. Interestingly, in macrophages different trends have been reported with regard to glucose oxidation. In particular, in M1 macrophages glucose is shifted towards lactate production and away from the TCA cycle [63], while the glucose flux through the PPP increases [55]. In contrast to M1 macrophages, M1 microglia utilize glucose through the TCA cycle and do not show any 
changes in the PPP. This highlights the fact that although macrophages and microglia share many characteristics, they also differ profoundly. In our study, we also report that similarly to macrophages [64], M1 microglia show increased glutamine oxidation. Based on the higher expression of Got1 in these cells, we speculate that the entrance of glutamine into the TCA cycle is catalyzed by GOT enzyme rather than GLUD. Interestingly, the reaction catalyzed by GOT could be highly important for M1 microglia as it also converts oxaloacetate, via the aspartateargininosuccinate shunt, to arginine [63]. Arginine can then be used for NO synthesis, which is a hallmark of the M1 state. Furthermore, a recent study in primary microglia highlights the role of glutamine in microglia polarization as it reported higher expression and activity of GS as well as an increased glutamine concentration in LPS-stimulated microglia [26]. Moreover, according to a recent study examining the metabolic plasticity of microglia, microglia seem to prefer glutamine as the most effective fuel, further favoring glutamine as a key nutrient for microglia [65]. Furthermore, manipulating glutamine metabolism has been shown to affect microglia polarization, as inhibition of GS led to enhanced inflammatory responses in microglia [26]. Considering the decreased expression of GS in the spinal cords of EAE mice [26], a model of inflammatory autoimmune disease, manipulating microglial glutamine metabolism might be able to dampen the inflammatory pathology in these mice. Taken together, we can propose that suppressing glutamine oxidation could constitute an alternative approach to reduce or abolish pro-inflammatory microglia polarization.

However, another goal would be not only to inhibit microglia from acquiring a pro-inflammatory phenotype, but also to shift them towards an anti-inflammatory phenotype in parallel. As we saw opposing regulation of both mitochondrial FAO and fatty acid synthesis in M1 and M2 microglia, we could speculate that targeting these pathways would yield such an effect. Moreover, fatty acid and lipid metabolism has been gaining more attention in the context of microglia polarization and function [66-71]. For example, lipoprotein lipase (LPL), an enzyme important for the release of fatty acids from TAG, has recently been identified as a feature of anti-inflammatory microglia [72] and was also found to be upregulated in disease-associated microglia [71]. Moreover, LPL inhibition has been linked to reduced expression of antiinflammatory and increased expression of pro-inflammatory markers, strengthening the idea of dependence of the microglia phenotype on lipid metabolism [72]. In our study, we proved increased FAO and fatty acid synthesis in the M2 microglia state, further strengthening the notion of the importance of fatty acid metabolism for an anti-inflammatory microglia phenotype. We could speculate that lipid metabolism, fatty acid synthesis and degradation in particular, might support a protective microglia phenotype, which could drive neuroprotection in neurodegenerative diseases. Furthermore, as we previously showed that peroxisomal FAO is a hallmark of M2 macrophages [73], and considering the novel roles of peroxisomes in 
immune cell functioning [74-77], it is of importance to also highlight this aspect of metabolism in microglia. In our study, we observed, in contrast to macrophages, no changes in peroxisomal FAO in polarized microglia. Nevertheless, genes encoding enzymes involved in peroxisomal FAO, but also mitochondrial FAO, were strongly suppressed in M1 microglia, indicating that a common transcriptional regulator might be involved. We hypothesize that peroxisome proliferator-activated receptor gamma coactivator (PGC)1a could be such regulator, as it has already been proven that PGC1a controls the expression of enzymes of both mitochondrial and peroxisomal $\beta$ oxidation [78]. Moreover, it has also been shown that BV-2 microglia downregulate PGC1a expression upon LPS stimulation and that its overexpression leads to reduced inflammatory responses of microglia upon LPS stimulation, favoring PGC1a as a potential regulator of M1 polarization [79]. Interestingly, another related coactivator, $\mathrm{PGC1} \beta$ has been assigned as a driver of increased mitochondrial biogenesis and FAO in M2 macrophages [19], and PCG1a might mirror such effects in the M1 state.

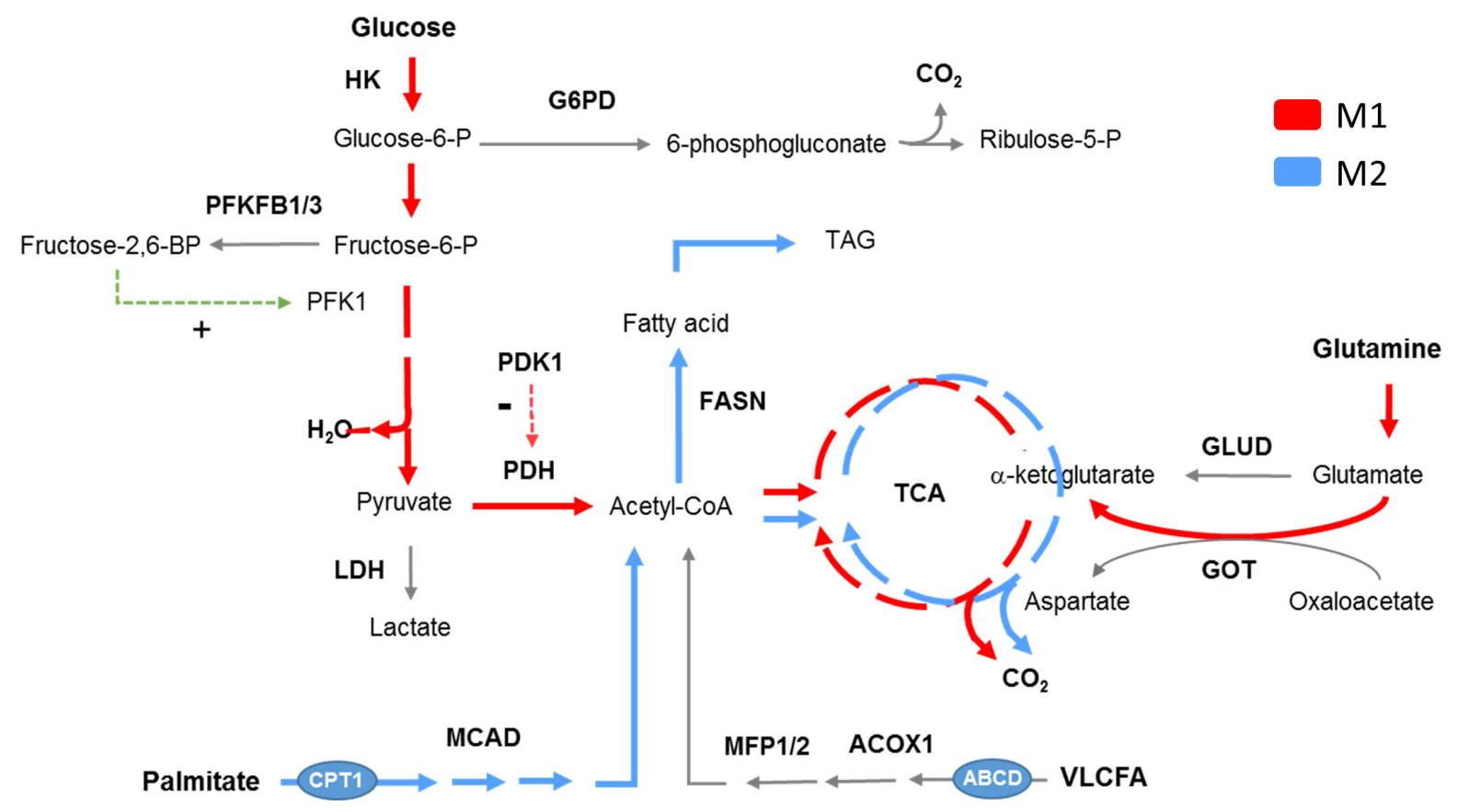

Figure 10. Metabolic features of pro- and anti-inflammatory microglia. Pro-inflammatory microglia are characterized by increased glucose and glutamine metabolism (glycolysis, glucose oxidation through the TCA cycle and glutamine oxidation), while anti-inflammatory microglia upregulate fatty acid metabolism (mitochondrial fatty acid oxidation and fatty acid synthesis). Metabolic pathways upregulated in proinflammatory, M1 microglia or anti-inflammatory, M2 microglia are represented by red and blue color, respectively. Enzymes and starting substrates are marked in bold. ABCD-ATP-binding cassette transporters of subfamily D; ACOX1, acyl-CoA oxidase 1; CPT1, carnitine palmitoyltransferase 1; FASN, fatty acid synthase; G6PD, glucose-6-phosphate dehydrogenase; GLUD, glutamate dehydrogenase; GOT, glutamate-oxaloacetate transaminase; HK, hexokinase; LDH, lactate dehydrogenase; MCAD, medium-chain acyl-CoA dehydrogenase; MFP, multifunctional protein; PDH, pyruvate dehydrogenase; PDK, pyruvate dehydrogenase kinase; PFKFB, phosphofructokinase/fructose biphosphatase; TAG, triacylglycerides; TCA, tricarboxylic acid cycle; VLCFA, very long chain fatty acids. 
Lastly, it should be emphasized that our experimental approach has limitations for several reasons. First, cultured microglia lose their specific signature when taken out of the brain environment $[5,80]$. In addition, pushing these cells to extreme states in vitro might not reflect the in vivo phenotypes that microglia adopt in pathological conditions [81]. Nevertheless, since studying microglia metabolism in the heterogeneous cellular environment of the brain is not possible, it was useful to reveal that cultured pro- and anti-inflammatory microglia display robust differences in fuel usage.

\section{CONCLUSIONS}

We thoroughly defined the different metabolic pathways employed by microglia polarized into a clear-cut pro- and anti-inflammatory state. Pro-inflammatory microglia exhibit increased glucose and glutamine metabolism and suppress both fatty acid oxidation and to a lesser extent fatty acid synthesis. On the other hand, anti-inflammatory microglia display changes only in fatty acid metabolism upregulating both fatty acid oxidation and fatty acid synthesis. We propose future research on the causal link between metabolism and microglia phenotype in health and disease, to eventually allow us to manipulate microglial metabolism in order to protect the brain.

\section{SUPPLEMENTARY MATERIALS}

The following supplementary materials are available online at https://doi.org/10.20900/immunometab20190002, Figure S1: Successful polarization of primary microglia towards an M1 and M2 phenotype.

\section{AUTHORS CONTRIBUTION}

IG designed the study, performed experiments, analyzed data and wrote the manuscript. MB and PC designed the study, supervised experiments and wrote the manuscript. SS helped perform and analyze experiments regarding metabolic assays. CC and CMV provided hPSC-derived microglia-like cells and commented on the manuscript. PG and $\mathrm{CV}$ assisted in the experimental setup and commented on the manuscript. PPVV helped perform and analyze experiments regarding metabolic assays and commented on the manuscript. We thank Benny Das, Carla De Legher and Inge Betz for excellent technical assistance.

\section{DISCLOSURE OF FUNDING SOURCES}

The funders had no role in study design, data collection and analysis, decision to publish, or preparation of the manuscript. 


\section{CONFLICT OF INTEREST}

The authors declare that there is no conflict of interest regarding the publication of this paper.

\section{ACKNOWLEDGEMENTS}

IG was supported by an ERAWEB fellowship. Research was supported by grants from KU Leuven (OT12/78 to MB), from ERA-Net Neuron (MICRO-MET to MB, PC, PG and CV), and from Merck Serono (to CV, PG, $\mathrm{PC}$ and $\mathrm{MB})$.

\section{REFERENCES}

1. Kierdorf K, Prinz M. Microglia in steady state. J Clin Invest. 2017;127(9):3201-9.

2. Prinz M, Priller J. Microglia and brain macrophages in the molecular age: from origin to neuropsychiatric disease. Nat Rev Neurosci. 2014;15(5):300-12.

3. Gentek R, Molawi K, Sieweke MH. Tissue macrophage identity and selfrenewal. Immunol Rev. 2014;262(1):56-73.

4. Gautier EL, Shay T, Miller J, Greter M, Jakubzick C, Ivanov S, et al. Geneexpression profiles and transcriptional regulatory pathways that underlie the identity and diversity of mouse tissue macrophages. Nat Immunol. 2012;13(11):1118-28.

5. Butovsky O, Jedrychowski MP, Moore CS, Cialic R, Lanser AJ, Gabriely G, et al. Identification of a unique TGF-beta-dependent molecular and functional signature in microglia. Nat Neurosci. 2014;17(1):131-43.

6. Nimmerjahn A, Kirchhoff F, Helmchen F. Resting microglial cells are highly dynamic surveillants of brain parenchyma in vivo. Science. 2005;308(5726):1314-8.

7. Chhor V, Le Charpentier T, Lebon S, Ore MV, Celador IL, Josserand J, et al. Characterization of phenotype markers and neuronotoxic potential of polarised primary microglia in vitro. Brain Behav Immun. 2013;32:70-85.

8. Tang Y, Le W. Differential Roles of M1 and M2 Microglia in Neurodegenerative Diseases. Mol Neurobiol. 2016;53(2):1181-94.

9. Meda L, Baron P, Prat E, Scarpini E, Scarlato G, Cassatella MA, et al. Proinflammatory profile of cytokine production by human monocytes and murine microglia stimulated with beta-amyloid[25-35]. J Neuroimmunol. 1999;93(1-2):45-52.

10. Rothwell NJ, Luheshi GN. Interleukin 1 in the brain: biology, pathology and therapeutic target. Trends Neurosci. 2000;23(12):618-25.

11. Halle A, Hornung V, Petzold GC, Stewart CR, Monks BG, Reinheckel T, et al. The NALP3 inflammasome is involved in the innate immune response to amyloid-beta. Nat Immunol. 2008;9(8):857-65.

12. Rossi C, Cusimano M, Zambito M, Finardi A, Capotondo A, Garcia-Manteiga JM, et al. Interleukin 4 modulates microglia homeostasis and attenuates the early slowly progressive phase of amyotrophic lateral sclerosis. Cell Death Dis. 2018;9(2):250. 
13. Spittau B. Interleukin 4-induced neuroprotection and regulation of microglia activation as a therapeutic approach in the MPTP model of Parkinson's disease. Neural Regen Res. 2017;12(9):1433-4.

14. Chao CC, Molitor TW, Hu S. Neuroprotective role of IL-4 against activated microglia. J Immunol. 1993;151(3):1473-81.

15. Amor S, Peferoen LA, Vogel DY, Breur M, van der Valk P, Baker D, et al. Inflammation in neurodegenerative diseases--an update. Immunology. 2014;142(2):151-66.

16. Fumagalli M, Lombardi M, Gressens P, Verderio C. How to reprogram microglia toward beneficial functions. Glia. 2018. doi. 10.1002/glia.23484

17. Borst K, Schwabenland M, Prinz M. Microglia metabolism in health and disease. Neurochem Int. 2018. doi. 10.1016/j.neuint.2018.11.006

18. Rodriguez-Prados JC, Traves PG, Cuenca J, Rico D, Aragones J, Martin-Sanz P, et al. Substrate fate in activated macrophages: a comparison between innate, classic, and alternative activation. J Immunol. 2010;185(1):605-14.

19. Vats D, Mukundan L, Odegaard JI, Zhang L, Smith KL, Morel CR, et al. Oxidative metabolism and PGC-1beta attenuate macrophage-mediated inflammation. Cell Metab. 2006;4(1):13-24.

20. Wang F, Zhang S, Jeon R, Vuckovic I, Jiang X, Lerman A, et al. Interferon Gamma Induces Reversible Metabolic Reprogramming of M1 Macrophages to Sustain Cell Viability and Pro-Inflammatory Activity. EBioMedicine. 2018. doi. 10.1016/j.ebiom.2018.02.009

21. Na YR, Je S, Seok SH. Metabolic features of macrophages in inflammatory diseases and cancer. Cancer Lett. 2018;413:46-58.

22. Voloboueva LA, Emery JF, Sun X, Giffard RG. Inflammatory response of microglial BV-2 cells includes a glycolytic shift and is modulated by mitochondrial glucose-regulated protein 75/mortalin. FEBS Lett. 2013;587(6):756-62.

23. Orihuela R, McPherson CA, Harry GJ. Microglial M1/M2 polarization and metabolic states. Br J Pharmacol. 2016;173(4):649-65.

24. Gimeno-Bayon J, Lopez-Lopez A, Rodriguez MJ, Mahy N. Glucose pathways adaptation supports acquisition of activated microglia phenotype. J Neurosci Res. 2014;92(6):723-31.

25. Holland R, McIntosh AL, Finucane OM, Mela V, Rubio-Araiz A, Timmons G, et al. Inflammatory microglia are glycolytic and iron retentive and typify the microglia in APP/PS1 mice. Brain Behav Immun. 2018;68:183-96.

26. Palmieri EM, Menga A, Lebrun A, Hooper DC, Butterfield DA, Mazzone M, et al. Blockade of Glutamine Synthetase Enhances Inflammatory Response in Microglial Cells. Antioxid Redox Signal. 2017;26(8):351-63.

27. Nakajima K, Kanamatsu T, Takezawa Y, Kohsaka S. Up-regulation of glutamine synthesis in microglia activated with endotoxin. Neurosci Lett. 2015;591:99-104.

28. Moss DW, Bates TE. Activation of murine microglial cell lines by lipopolysaccharide and interferon-gamma causes NO-mediated decreases in mitochondrial and cellular function. Eur J Neurosci. 2001;13(3):529-38. 
29. Chenais B, Morjani H, Drapier JC. Impact of endogenous nitric oxide on microglial cell energy metabolism and labile iron pool. J Neurochem. 2002;81(3):615-23.

30. Ulland TK, Song WM, Huang SC, Ulrich JD, Sergushichev A, Beatty WL, et al. TREM2 Maintains Microglial Metabolic Fitness in Alzheimer's Disease. Cell. 2017;170(4):649-63 e13.

31. Li Y, Lu B, Sheng L, Zhu Z, Sun H, Zhou Y, et al. Hexokinase 2-dependent hyperglycolysis driving microglial activation contributes to ischemic brain injury. J Neurochem. 2018;144(2):186-200.

32. Locatelli G, Theodorou D, Kendirli A, Jordao MJC, Staszewski O, Phulphagar K, et al. Mononuclear phagocytes locally specify and adapt their phenotype in a multiple sclerosis model. Nat Neurosci. 2018;21(9):1196-208.

33. Flowers A, Bell-Temin H, Jalloh A, Stevens SM, Bickford PC. Proteomic anaysis of aged microglia: shifts in transcription, bioenergetics, and nutrient response. J Neuroinflammation. 2017;14(1):96.

34. Yao J, Chen S, Mao Z, Cadenas E, Brinton RD. 2-Deoxy-D-glucose treatment induces ketogenesis, sustains mitochondrial function, and reduces pathology in female mouse model of Alzheimer's disease. PLoS One. 2011;6(7):e21788.

35. Duan W, Mattson MP. Dietary restriction and 2-deoxyglucose administration improve behavioral outcome and reduce degeneration of dopaminergic neurons in models of Parkinson's disease. J Neurosci Res. 1999;57(2):195-206.

36. Garriga-Canut M, Schoenike B, Qazi R, Bergendahl K, Daley TJ, Pfender RM, et al. 2-Deoxy-D-glucose reduces epilepsy progression by NRSF-CtBPdependent metabolic regulation of chromatin structure. Nat Neurosci. 2006;9(11):1382-7.

37. Shriver LP, Manchester M. Inhibition of fatty acid metabolism ameliorates disease activity in an animal model of multiple sclerosis. Sci Rep. 2011;1:79.

38. Claes C, Van Den Daele J, Boon R, Schouteden S, Colombo A, Monasor LS, et al. Human stem cell-derived monocytes and microglia-like cells reveal impaired amyloid plaque clearance upon heterozygous or homozygous loss of TREM2. Alzheimers Dement. 2018. DOI: https://doi.org/10.1016/j.jalz.2018.09.006

39. Verheijden S, Beckers L, Casazza A, Butovsky O, Mazzone M, Baes M. Identification of a chronic non-neurodegenerative microglia activation state in a mouse model of peroxisomal beta-oxidation deficiency. Glia. 2015;63(9):1606-20.

40. Beckers L, Stroobants S, D'Hooge R, Baes M. Neuronal Dysfunction and Behavioral Abnormalities Are Evoked by Neural Cells and Aggravated by Inflammatory Microglia in Peroxisomal beta-Oxidation Deficiency. Front Cell Neurosci. 2018;12:136.

41. Livak KJ, Schmittgen TD. Analysis of relative gene expression data using real-time quantitative PCR and the 2(-Delta Delta C(T)) Method. Methods. 2001;25(4):402-8.

42. Van Veldhoven PP, Van Rompuy P, Fransen M, De Bethune B, Mannaerts GP. Large-scale purification and further characterization of rat pristanoyl-CoA oxidase. Eur J Biochem. 1994;222(3):795-801. 
43. Schoors S, Bruning U, Missiaen R, Queiroz KC, Borgers G, Elia I, et al. Fatty acid carbon is essential for dNTP synthesis in endothelial cells. Nature. 2015;520(7546):192-7.

44. Veys K, Alvarado-Diaz A, De Bock K. Measuring Glycolytic and Mitochondrial Fluxes in Endothelial Cells Using Radioactive Tracers. Methods Mol Biol. 2019;1862:121-36.

45. Ashcroft SJ, Weerasinghe LC, Bassett JM, Randle PJ. The pentose cycle and insulin release in mouse pancreatic islets. Biochem J. 1972;126(3):525-32.

46. Vanhove G, Van Veldhoven PP, Vanhoutte F, Parmentier G, Eyssen HJ, Mannaerts GP. Mitochondrial and peroxisomal beta oxidation of the branched chain fatty acid 2-methylpalmitate in rat liver. J Biol Chem. 1991;266(36):24670-5.

47. Van Veldhoven PP, Huang S, Eyssen HJ, Mannaerts GP. The deficient degradation of synthetic 2- and 3-methyl-branched fatty acids in fibroblasts from patients with peroxisomal disorders. J Inherit Metab Dis. 1993;16(2):381-91.

48. Esquenet M, Swinnen JV, Van Veldhoven PP, Denef C, Heyns W, Verhoeven G. Retinoids stimulate lipid synthesis and accumulation in LNCaP prostatic adenocarcinoma cells. Mol Cell Endocrinol. 1997;136(1):37-46.

49. Van Veldhoven PP, Bell RM. Effect of harvesting methods, growth conditions and growth phase on diacylglycerol levels in cultured human adherent cells. Biochim Biophys Acta. 1988;959(2):185-96.

50. Van Veldhoven PP, Mannaerts GP. Inorganic and organic phosphate measurements in the nanomolar range. Anal Biochem. 1987;161(1):45-8.

51. Ros S, Schulze A. Balancing glycolytic flux: the role of 6-phosphofructo-2kinase/fructose 2,6-bisphosphatases in cancer metabolism. Cancer Metab. 2013;1(1):8.

52. Huang SC, Smith AM, Everts B, Colonna M, Pearce EL, Schilling JD, et al. Metabolic Reprogramming Mediated by the mTORC2-IRF4 Signaling Axis Is Essential for Macrophage Alternative Activation. Immunity. 2016;45(4):817-30.

53. Tan Z, Xie N, Cui H, Moellering DR, Abraham E, Thannickal VJ, et al. Pyruvate dehydrogenase kinase 1 participates in macrophage polarization via regulating glucose metabolism. J Immunol. 2015;194(12):6082-9.

54. Hothersall JS, Baquer N, Greenbaum AL, McLean P. Alternative pathways of glucose utilization in brain. Changes in the pattern of glucose utilization in brain during development and the effect of phenazine methosulfate on the integration of metabolic routes. Arch Biochem Biophys. 1979;198(2):478-92.

55. Haschemi A, Kosma P, Gille L, Evans CR, Burant CF, Starkl P, et al. The sedoheptulose kinase CARKL directs macrophage polarization through control of glucose metabolism. Cell Metab. 2012;15(6):813-26.

56. Van Veldhoven PP. Biochemistry and genetics of inherited disorders of peroxisomal fatty acid metabolism. J Lipid Res. 2010;51(10):2863-95.

57. Moore SA, Hurt E, Yoder E, Sprecher H, Spector AA. Docosahexaenoic acid synthesis in human skin fibroblasts involves peroxisomal retroconversion of tetracosahexaenoic acid. J Lipid Res. 1995;36(11):2433-43. 
58. Norden DM, Trojanowski PJ, Villanueva E, Navarro E, Godbout JP. Sequential activation of microglia and astrocyte cytokine expression precedes increased Iba-1 or GFAP immunoreactivity following systemic immune challenge. Glia. 2016;64(2):300-16.

59. Shen Y, Kapfhamer D, Minnella AM, Kim JE, Won SJ, Chen Y, et al. Bioenergetic state regulates innate inflammatory responses through the transcriptional co-repressor CtBP. Nat Commun. 2017;8(1):624.

60. Ghosh S, Castillo E, Frias ES, Swanson RA. Bioenergetic regulation of microglia. Glia. 2018;66(6):1200-12.

61. Schoors S, Cantelmo AR, Georgiadou M, Stapor P, Wang X, Quaegebeur A, et al. Incomplete and transitory decrease of glycolysis: a new paradigm for antiangiogenic therapy? Cell Cycle. 2014;13(1):16-22.

62. Tawakol A, Singh P, Mojena M, Pimentel-Santillana M, Emami H, MacNabb M, et al. HIF-1alpha and PFKFB3 Mediate a Tight Relationship Between Proinflammatory Activation and Anerobic Metabolism in Atherosclerotic Macrophages. Arterioscler Thromb Vasc Biol. 2015;35(6):1463-71.

63. Jha AK, Huang SC, Sergushichev A, Lampropoulou V, Ivanova Y, Loginicheva E, et al. Network integration of parallel metabolic and transcriptional data reveals metabolic modules that regulate macrophage polarization. Immunity. 2015;42(3):419-30.

64. Tannahill GM, Curtis AM, Adamik J, Palsson-McDermott EM, McGettrick AF, Goel G, et al. Succinate is an inflammatory signal that induces IL-1beta through HIF-1alpha. Nature. 2013;496(7444):238-42.

65. Nagy AM, Fekete R, Horvath G, Koncsos G, Kriston C, Sebestyen A, et al. Versatility of microglial bioenergetic machinery under starving conditions. Biochim Biophys Acta Bioenerg. 2018;1859(3):201-14.

66. Karasinska JM, de Haan W, Franciosi S, Ruddle P, Fan J, Kruit JK, et al. ABCA1 influences neuroinflammation and neuronal death. Neurobiol Dis. 2013;54:445-55.

67. Gao Y, Vidal-Itriago A, Kalsbeek MJ, Layritz C, Garcia-Caceres C, Tom RZ, et al. Lipoprotein Lipase Maintains Microglial Innate Immunity in Obesity. Cell Rep. 2017;20(13):3034-42.

68. Khatchadourian A, Bourque SD, Richard VR, Titorenko VI, Maysinger D. Dynamics and regulation of lipid droplet formation in lipopolysaccharide (LPS)-stimulated microglia. Biochim Biophys Acta. 2012;1821(4):607-17.

69. $\mathrm{Hu} \mathrm{X}, \mathrm{Xu} \mathrm{B}, \mathrm{Ge}$ W. The Role of Lipid Bodies in the Microglial Aging Process and Related Diseases. Neurochem Res. 2017;42(11):3140-8.

70. Button EB, Mitchell AS, Domingos MM, Chung JH, Bradley RM, Hashemi A, et al. Microglial cell activation increases saturated and decreases monounsaturated fatty acid content, but both lipid species are proinflammatory. Lipids. 2014;49(4):305-16.

71. Keren-Shaul H, Spinrad A, Weiner A, Matcovitch-Natan O, Dvir-Szternfeld R, Ulland TK, et al. A Unique Microglia Type Associated with Restricting Development of Alzheimer's Disease. Cell. 2017;169(7):1276-90 e17.

72. Bruce KD, Gorkhali S, Given K, Coates AM, Boyle KE, Macklin WB, et al. Lipoprotein Lipase Is a Feature of Alternatively-Activated Microglia and 
May Facilitate Lipid Uptake in the CNS During Demyelination. Front Mol Neurosci. 2018;11:57.

73. Geric I, Tyurina YY, Krysko O, Krysko DV, De Schryver E, Kagan VE, et al. Lipid homeostasis and inflammatory activation are disturbed in classically activated macrophages with peroxisomal beta-oxidation deficiency. Immunology. 2018 Mar;153(3):342-56. doi: 10.1111/imm.12844

74. Di Cara F, Sheshachalam A, Braverman NE, Rachubinski RA, Simmonds AJ. Peroxisome-Mediated Metabolism Is Required for Immune Response to Microbial Infection. Immunity. 2017;47(1):93-106 e7.

75. Vijayan V, Srinu T, Karnati S, Garikapati V, Linke M, Kamalyan L, et al. A New Immunomodulatory Role for Peroxisomes in Macrophages Activated by the TLR4 Ligand Lipopolysaccharide. J Immunol. 2017;198(6):2414-25.

76. Raas Q, Saih FE, Gondcaille C, Trompier D, Hamon Y, Leoni V, et al. A microglial cell model for acyl-CoA oxidase 1 deficiency. Biochim Biophys Acta Mol Cell Biol Lipids. 2019 April;1864(4):567-76

77. Weinhofer I, Zierfuss B, Hametner S, Wagner M, Popitsch N, Machacek C, et al. Impaired plasticity of macrophages in X-linked adrenoleukodystrophy. Brain. 2018 Aug 1;141(8):2329-42. doi: 10.1093/brain/awy127

78. Huang TY, Zheng D, Houmard JA, Brault JJ, Hickner RC, Cortright RN. Overexpression of PGC-1alpha increases peroxisomal activity and mitochondrial fatty acid oxidation in human primary myotubes. Am J Physiol Endocrinol Metab. 2017;312(4):E253-63.

79. Yang X, Xu S, Qian Y, Xiao Q. Resveratrol regulates microglia M1/M2 polarization via PGC-1alpha in conditions of neuroinflammatory injury. Brain Behav Immun. 2017;64:162-72.

80. Gosselin D, Link VM, Romanoski CE, Fonseca GJ, Eichenfield DZ, Spann NJ, et al. Environment drives selection and function of enhancers controlling tissue-specific macrophage identities. Cell. 2014;159(6):1327-40.

81. Ransohoff RM. A polarizing question: do M1 and M2 microglia exist? Nat Neurosci. 2016;19(8):987-91.

How to cite this article:

Geric I, Schoors S, Claes C, Gressens P, Verderio, C, Verfaillie CM, Van Veldhoven PP, Carmeliet P, Baes M. Metabolic Reprogramming during Microglia Activation. Immunometabolism. 2019;1:e190002. https://doi.org/10.20900/immunometab20190002 